Article

\title{
Statistical Analysis of 1996-2017 Ozone Profile Data Obtained by Ground-Based Microwave Radiometry
}

\author{
Konstantin P. Gaikovich ${ }^{1, *(\mathbb{D} \text {, Elena P. Kropotkina }}{ }^{2}$ and Sergey B. Rozanov ${ }^{2,3}$ \\ 1 Institute for Physics of Microstructure RAS, 603950 Nizhny Novgorod, Russia \\ 2 P.N. Lebedev Physical Institute RAS, 119991 Moscow, Russia; ekrop40@mail.ru (E.P.K.); \\ sergroz_fian@mail.ru (S.B.R.) \\ 3 Russian Metrological Institute of Technical Physics and Radio Engineering, 141570 Mendeleevo, \\ Moscow Region, Russia \\ * Correspondence: gaikovich@mail.ru
}

Received: 13 August 2020; Accepted: 13 October 2020; Published: 15 October 2020

\begin{abstract}
Trends in the ozone layer remain among the major problems of the atmosphere physics; thus, results of measurements of the ozone altitude distribution (profile), carried out in the same place and via the same method, are very important. This paper presents the results of the statistical analysis of ensembles of ozone profiles obtained from ground-based microwave radiometry data acquired at the P.N. Lebedev Physical Institute over a period of two decades (1996-2017). The data collected show the significant difference between monthly mean statistical parameters of ozone profiles of the decades 1996-2006 and 2007-2017. The main and unexpected result is the drastic decrease in monthly root-mean-square $(\mathrm{rms})$ variances of ozone profiles over Moscow above $30 \mathrm{~km}$ in cold months of the decade 2007-2017 (if compared to the variances in the decade 1996-2006) with the maximum fall by $46 \%$ at $39 \mathrm{~km}$ in February monthly mean variances. The decade change of variances obtained by averaging over all nine months in the analysis (from September to May) has the same decrease with maximum fall by $25 \%$ at $38 \mathrm{~km}$. Additionally, significant decade changes were revealed in other monthly mean statistical parameters: probability density of ozone profile variances, inter-altitude covariance and correlation functions, and time covariance and correlation-as well as their frequency spectra. The decade change of the ozone profile obtained by averaging over the nine months appeared much less significant: the decrease by $5.7 \%$ at the altitude of $19 \mathrm{~km}$ (with $1.5 \%$ sampling error), minor decrease by $2.6 \%$ (with sampling error $1.5 \%$ ) in the profile maximum at $37 \mathrm{~km}$, and increases of $1.7 \%$ at $28 \mathrm{~km}$ and $2.5 \%$ at $47 \mathrm{~km}$ (with sampling errors $1.7 \%$ ) - lower and higher of this maximum. In addition to that, the corresponding averaged mean total column (integral) ozone content above $20 \mathrm{~km}$ remained practically unchanged: $4.61 \mathrm{~g} / \mathrm{m}^{2}$ for decade $1996-2006$ as compared to $4.58 \mathrm{~g} / \mathrm{m}^{2}$ for 2007-2017. Possible explanations of revealed offsets are proposed and discussed.
\end{abstract}

Keywords: remote sensing; ozone profile; microwave radiometry; inverse problems; statistical analysis

\section{Introduction}

The depletion of the ozone layer, which protects life on the Earth from the harmful solar UVB radiation by anthropogenic chlorine and bromine, remains among the major problems. As mentioned in [1], the Montreal Protocol has been successful, and the decline in ozone in the upper stratosphere stopped around in 1996-1997 [2], and in 2014, the increase in ozone was observed; however, it was statistically significant only in the upper stratosphere (around $42 \mathrm{~km}$ or $2 \mathrm{hPa}$ ), but not at lower levels, nor for total ozone columns [2]. More recent data of satellite and ground-based measurements of ozone profiles published in recent years (see in a comprehensive review [2]) demonstrate significant 
ozone increases in the upper stratosphere, between 35 and $48 \mathrm{~km}$ altitude ( 5 and $1 \mathrm{hPa}$ ). Closer to $2 \mathrm{hPa}(42 \mathrm{~km})$, the ozone was increasing by about $1.5 \%$ per decade in the tropics, and by 2 to $2.5 \%$ per

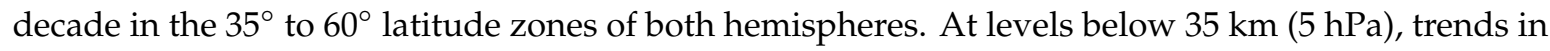
the ozone layer from 2000 to 2016 were smaller and not statistically significant. This study confirms positive trends of the upper stratospheric ozone reported in [1]. The observed trends of profiles are consistent with expectations from chemistry climate model simulations.

In recent years, multiple studies of the ozone layer were related to the influence of planetary waves, polar stratospheric vortex, and sudden stratospheric warmings on the formation of ozone fields and other trace gases in the atmosphere [3-5]. The quantitative study was applied to determine numerical models of the atmosphere dynamics, chemistry, and thermal regime that describe the effects of stratospheric warmings on stratosphere ozone and other trace gases $[3,4]$. Such models are developed on the basis of experimental data on changes in the ozone vertical distribution not only in the upper and lower stratosphere, where ozone is the most sensitive to the anthropogenic pollution with chlorofluorocarbons [1], but also in the middle stratosphere layers (10 mbar, height $30 \mathrm{~km})$, where the ozone is expected to decrease due to the higher nitrogen content in the stratosphere [6]. To date, the origin of the ozone depletion has not been sufficiently studied, and it is sometimes unpredictable, such as "the Arctic ozone hole" in the spring of 2011 [7]. So, as mentioned in [2], a thorough analysis of possible drifts and differences between various data sources is still required. Ongoing quality observations from multiple independent platforms are the key for verifying that recovery of the ozone layer continues as expected [2].

In view of these studies, the results presented herein of the statistical analysis of the two-decade (1996-2017) ensemble of profiles of the ozone volume mixing ratio obtained in the same place and with the same method are very important. The geographical position of Moscow allows the observation of air masses of very different origins including, in particular, the air of the polar stratospheric vortex when it appears over Moscow that leads to strong variations of the ozone profile, especially in periods of violent stratospheric disturbances and sudden midwinter stratospheric warmings that occurred in the past two decades. Additionally, strong ozone variations can be related to planetary waves.

\section{Equipment, Measurements, and Data Processing}

Our study is based on data of the ground-based remote sensing of the atmospheric ozone at millimeter waves (microwaves), which is an effective way to study the ozone vertical distribution [8-12]. The monitoring of brightness temperatures of the atmospheric ozone thermal emission at frequencies of its spectral line centered at $142.175 \mathrm{GHz}$ was carried out at the P.N. Lebedev Physical Institute of the

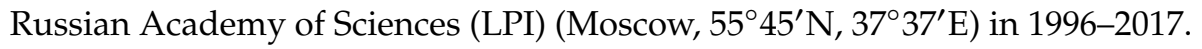

Amplitude-switching heterodyne receiver of the LPI ozone spectrometer (ozonometer) is based on the planar Schottky diode mixer. Single sideband receiver noise temperature is about $1500 \mathrm{~K}$ without cooling and about $700 \mathrm{~K}$ under liquid nitrogen cooling. Typically, room temperature operation was used for regular day-time ozone measurements in 1996-2017. Two filter-bank spectrum analyzers were used (80 spectral channels in the frequency bandwidth of $283 \mathrm{MHz}$ from 1996 to 2007 [13] and 96 channels in the bandwidth of $475 \mathrm{MHz}$ since 2007 [14]). The width of their channels increased in five steps from $0.2 / 0.1 \mathrm{MHz}$ in the ozone line center to $20 \mathrm{MHz}$ at wings, so the devices were adapted to the line contour [9,10]. In 2005-2006, ozone observations were carried out with both spectrum analyzers to compare spectra and retrieved ozone profiles obtained with the filter-banks. This provided continuity of ozone measurements in two decades. The Gaussian antenna pattern of the ozonometer is of $1.5^{\circ} \mathrm{HPFW}$ without side-lobes above $-30 \mathrm{~dB}$. Brightness temperature of the atmosphere measured in broadband $(\sim 0.6 \mathrm{GHz})$ channel of the instrument was used to determine tropospheric loss due to molecular oxygen and water. Two external blackbody loads (at room and liquid nitrogen temperatures) were used for calibration of the ozonometer.

All measurements were carried out in the daytime. Duration of the measurements was $1-3 \mathrm{~h}$ depending on weather conditions. In fact, our ground-based microwave ozone measurements (unlike 
lidar measurements) are possible for wide weather conditions (haze, fog, clouds, snow) except for drizzle and rain when tropospheric loss for zenith angle of $60^{\circ}$ was more than approximately $5 \mathrm{~dB}$. Mean number of measurements used for the statistical analysis was 15-20 per month (typically, all working days except for ones with bad weather). Data of night-time observations were too rare for the statistical analysis (about 50 nights) and were not included in the paper.

Data processing included recalculating unit $200 \mathrm{~s}$ ozone spectra outside the troposphere (multiplying them by the tropospheric loss, corresponding systematic errors of 1-1.5\% were vanished in the differential version of the presented below algorithm) and averaging the spectra (about 20 ones for good weather) to increase the signal-to-noise ratio.

This microwave technique gives day-and-night measurements of the ozone profile at altitudes from approximately 15 to $70 \mathrm{~km}$ (up to $100 \mathrm{~km}$ at night) [10] and is the most suitable one for long-term ground-based monitoring of the ozone profile in the stratosphere and lower mesosphere.

The LPI microwave ozonometer that provides data of systematic measurements of the vertical ozone profile in the stratosphere was included in the global ozonometric network in the framework of international programs DYANA, CRISTA/MAHRSI, and SOLVE. As an important milestone in the development of this network, the first simultaneous microwave observations of the ozonosphere from different points were carried out at the LPI and at Onsala Space Observatory (OSO, Sweden) in 1988-1990 [15].

The statistical analysis is based on the radiometric ozone monitoring in 1996-2017 (2096 vertical ozone profiles over Moscow with $1 \mathrm{~km}$ height step). The data were obtained for nine months from September to May, including 1169 profiles in the 1996-2006 decade and 927 ones in the 2007-2017 decade. These long measurements carried out in the same place make it possible to study long-term changes in ozonosphere. Diurnal variations are absent in the data obtained approximately in the same hours of daytime; however, the effect of seasonal variations on statistical parameters was studied and taken into account. Nine data sets-for September (59 elements), October (134), November (248), December (337), January (374), February (357), March (341), April (184), and May (62) (for autumn, winter and spring seasons)-were extracted from the data. Summer months were not included in the analysis (measurements were sparse and not enough informative because of very low level of summer ozone variations). To study long-term changes, the data extracted for each month were divided into two ensembles of 1996-2006 and 2007-2017 decades.

Ozone profile retrieval from measured spectra of brightness temperatures is based on solving the corresponding nonlinear integral equation with the iterative algorithm developed in [16] an successfully applied in $[9,10]$, where at each step of iterations the corresponding Fredholm integral equation of the 1st kind is solved with the Tikhonov's method of generalized discrepancy [17] that uses general a priori information on the smoothness of the exact solution. Unlike methods based on the "common wisdom" regularization [18-20] or the formal statistical regularization [21] of this ill-posed problem, this method is mathematically consistent and provides the convergence to the exact solution in the Sobolev's functional class $\mathrm{W}_{2}^{1}$ (see details in Appendix A). This approach was firstly proposed in the inverse problem of limb-viewing satellite microwave ozone profiling [22,23]. In this paper, we demonstrate results of this method in the study of long-term offsets in mean ozone profiles, their inter-altitude, and time variations.

\section{Statistical Analysis}

\subsection{Statistical Parameters and Method of Errors Correction}

Based on above-described data ensembles of ozone profiles $U(h)$ retrieved by solving algorithm (A9), see Appendix A, various statistical parameters were calculated: mean ozone profiles $\langle U(h)\rangle$, profiles of variances $\sigma^{2}(h)=<[U(h)-<U(h)>]^{2}>$ and rms deviations of ozone profile $\sigma(h)$, probability density of ozone profile variations $P(U(h)-\langle U(h)>$ ), inter-altitude covariance functions $B\left(h_{1}, h_{2}\right)=<\left[U\left(h_{1}\right)-<U\left(h_{1}\right)>\right]\left[\left(U\left(h_{2}\right)-<U\left(h_{2}\right)>\right]>\right.$, and inter-altitude correlation functions 
$R\left(h_{1}, h_{2}\right)=B\left(h_{1}, h_{2}\right) / \sqrt{B\left(h_{1}, h_{1}\right) B\left(h_{2}, h_{2}\right)}$. For time intervals $\tau$ much less than the typical time of seasonal variations, time covariance functions $B_{t}(h, \tau)=<[U(h, t)-<U(h)>][(U(h, t+\tau)-<U(h)>$ ] >, time correlation functions $R_{t}(h, \tau)=B_{t}(h, \tau) / \sigma^{2}(h)=B_{t}(h, \tau) / B(h, h)$, and their frequency spectra $S_{B}(h, f)=\int_{\Delta \tau} B_{t}(h, \tau) \exp (-2 \pi i f \tau) d \tau, S_{R}(h, f)=\int_{\Delta f} R_{t}(h, \tau) \exp (-2 \pi i f \tau) d \tau$ were determined. Available dimensions of data ensembles provided a high enough accuracy of these estimations.

It is worth mentioning that results of the numerical simulation [9,10] obtained for various simulated (typical and extreme) profiles gave the estimation of the retrieval accuracy in C-metric, but this simulation was unsuitable to obtain their statistical estimations. Now it appears possible to realize the numerical simulation according the steps (a)-(e) of the scheme described in the Appendix A after the formula (A11), where ensembles of the retrieved ozone profiles are used as preset ones that give us the set $\left\{U_{2}(h)\right\}$ of secondary retrieved profiles.

Let us write these profiles as sum $U_{2}(h)=U(h)+\Delta U(h)+\delta U(h)$ where $\Delta U(h)$ is the systematic error and $\delta U(h)$ is random error with zero mean value. Then, one can obtain statistical parameters of the retrieval errors of the method itself-systematic errors $\Delta U(h)=<U_{2}(h)-U(h)>$ and the dispersion of random errors $\sigma_{\delta U}^{2}(h)=<\left[U_{2}(h)-U(h)-\Delta U(h)\right]^{2}>$. Here, we propose to use these parameters to clear (correct) some statistical parameters of initially retrieved ozone profiles $U(h)$ from the methodical errors.

It is obvious that because of measurement and algorithm errors (systematic errors $\Delta U_{0}(h)$ and random errors $\delta U_{0}(h)$ with zero mean value), the retrieved ozone profiles $U(h)$ differ from corresponding true profiles $U_{0}(h)$, namely, $U(h)=U_{0}(h)+\Delta U_{0}(h)+\delta U_{0}(h)$, where parameters $\Delta U_{0}(h), \delta U_{0}(h)$ are unknown, and it leads to unknown distortions of true statistical parameters when they are estimated from ensembles of the retrieved profiles $U(h)$ :

$$
\begin{gathered}
<U(h)>=<U_{0}(h)>+\Delta U_{0}(h), \\
B\left(h_{1}, h_{2}\right)=<\left(U\left(h_{1}\right)-<U\left(h_{1}\right)>\right)\left(U\left(h_{2}\right)-<U\left(h_{2}\right)>\right)> \\
=<\left[U_{0}\left(h_{1}\right)-<U_{0}\left(h_{1}\right)>+\delta U_{0}\left(h_{1}\right)\right]\left[U_{0}\left(h_{2}\right)-<U_{0}\left(h_{2}\right)>+\delta U_{0}\left(h_{2}\right)\right]> \\
=B_{0}\left(h_{1}, h_{2}\right)+B_{U_{0} \delta U_{0}}\left(h_{1}, h_{2}\right)+B_{U_{0}} \delta U_{0}\left(h_{2}, h_{1}\right)+B_{\delta U_{0}}\left(h_{1}, h_{2}\right), \\
\sigma^{2}(h)=B(h, h)=\sigma_{0}^{2}(h)+2 B_{U_{0} \delta U_{0}}(h, h)+\sigma_{\delta U_{0}}^{2}(h), \\
B_{t}(h, \tau)=<\left[U_{0}(h, t)-<U_{0}(h, t)>+\delta U_{0}(h, t)\right]\left[\left(U_{0}(h, t+\tau)-<U_{0}(h, t+\tau)>+\delta U_{0}(h, t+\tau)\right]>\right. \\
=B_{t 0}(h, \tau)+B_{\delta U_{0}}(h, h) \delta(\tau),
\end{gathered}
$$

where $\delta(\tau)=1$ at $\tau=0 ; \delta(\tau)=0$ at $\tau \neq 0$.

In (4), it was taken into account that the retrieval errors are uncorrelated to ozone profiles as well as their errors obtained in different days; therefore, the time covariance of the retrieved profiles is a correct estimation of the true covariance function at $\tau \neq 0$-i.e., $B_{t 0}(h, \tau)=B_{t}(h, \tau)$.

From (1)-(4), one can express true statistical parameters for the profile ensemble $\left\{U_{0}\right\}$ via those obtained from the retrieved profiles $\{U\}$ :

$$
\begin{gathered}
<U_{0}(h)>=<U(h)>-\Delta U_{0}(h), \\
B_{0}\left(h_{1}, h_{2}\right)=B\left(h_{1}, h_{2}\right)-B_{U_{0} \delta U_{0}}\left(h_{1}, h_{2}\right)-B_{U_{0} \delta U_{0}}\left(h_{2}, h_{1}\right)-B_{\delta U_{0}}\left(h_{1}, h_{2}\right), \\
\sigma_{0}^{2}(h)=\sigma^{2}(h)-2 B_{U_{0} \delta U_{0}}(h, h)-\sigma_{\delta U_{0}}^{2}(h), \\
B_{t 0}(h, \tau)=B_{t}(h, \tau)-B_{\delta U_{0}}(h, h) \delta(\tau) .
\end{gathered}
$$

As it is possible to see, there are unknown differences between obtained and true statistical parameters because necessary statistical parameters $\Delta U_{0}(h), B_{U_{0} \delta U_{0}}\left(h_{1}, h_{2}\right)+$ $B_{U_{0} \delta U_{0}}\left(h_{2}, h_{1}\right), B_{\delta U_{0}}\left(h_{1}, h_{2}\right)$ of retrieval errors are unknown. However, realistic estimates of these parameters can be obtained in the numerical simulation using elements of ensembles of retrieved profiles $\{U\}$ as preset profiles that should be retrieved according to the above-described scheme 
(calculation of corresponding spectra of brightness temperature, adding normally distributed random errors with the $r m s$ value $\delta T_{B}=0.05 \mathrm{~K}$ of measurement errors, solving inverse problems, and comparing preset and retrieved profiles). As a result, we obtained the set of secondary retrieved profiles $\left\{U_{2}\right\}$. Then, it is possible to determine the same statistical parameters for $\left\{U_{2}\right\}$ as for $\{U\}$ :

$$
\begin{gathered}
<U_{2}(h)>=<U(h)>+\Delta U(h) \\
B_{2}\left(h_{1}, h_{2}\right)=B\left(h_{1}, h_{2}\right)+B_{U \delta U}\left(h_{1}, h_{2}\right)+B_{U \delta U}\left(h_{2}, h_{1}\right)+B_{\delta U,}\left(h_{1}, h_{2}\right) \\
B_{t 2}(h, \tau)=B_{t}(h, \tau)+B_{\delta U}(h, h) \delta(\tau)
\end{gathered}
$$

From expressions analogous to (5) and (6), statistical parameters $\Delta U(h), B_{U \delta U}\left(h_{1}, h_{2}\right)+$ $B_{U \delta U}\left(h_{2}, h_{1}\right), B_{\delta U}\left(h_{1}, h_{2}\right)$ of the secondary retrieved profiles $\left\{U_{2}\right\}$ can be determined. Then, we assume that these parameters are equal to corresponding error parameters of profiles $\{U\}$, retrieved by experimental data:

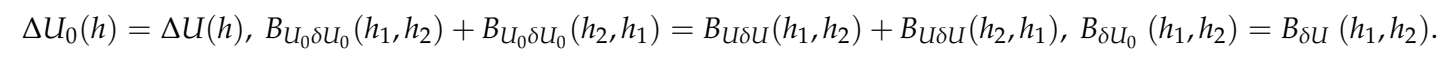

This enables to clear some of the above-mentioned statistical parameters extracted from retrieved ozone profiles $\{U\}$ from errors of retrieval. Finally, we have all the necessary formulas to estimate "true" statistical parameters of ozone by data of radiometric remote sensing:

$$
\begin{gathered}
<U_{0}(h)>=<U(h)>-\Delta U(h), \\
B_{0}\left(h_{1}, h_{2}\right)=B\left(h_{1}, h_{2}\right)-B_{U \delta U}\left(h_{1}, h_{2}\right)-B_{U \delta U}\left(h_{2}, h_{1}\right)-B_{\delta U}\left(h_{1}, h_{2}\right), \\
\sigma_{0}^{2}(h)=B_{0}(h, h)=\sigma^{2}(h)-2 B_{U \delta U}(h, h)-\sigma_{\delta U}^{2}(h), \\
R_{0}\left(h_{1}, h_{2}\right)=B_{0}\left(h_{1}, h_{2}\right) / \sqrt{B_{0}\left(h_{1}, h_{1}\right) B_{0}\left(h_{2}, h_{2}\right),} \\
B_{t 0}(h, \tau)=B_{t}(h, \tau)-B_{\delta U}(h, h) \delta(\tau), \\
R_{t 0}(h, \tau)=B_{t 0}(h, \tau) / B_{t 0}(h, 0), \\
S_{B 0}(h, f)=\int_{\Delta \tau} B_{t 0}(h, \tau) \exp (-2 \pi i f \tau) d \tau, \\
S_{R 0}(h, f)=\int_{\Delta \tau} R_{t 0}(h, \tau) \exp (-2 \pi i f \tau) d \tau .
\end{gathered}
$$

Of course, these "cleared" parameters can differ from those of real true profiles; however, these estimations should be realistic at least, where the retrieval errors are much less than $r m s$ variations of ozone profiles (at heights of $20-50 \mathrm{~km}$ ). In any case, it should be a useful real correction of methodical errors.

\subsection{Statistical Parameters of Retrieval Errors}

Errors of the ozone profile retrieval can be described by the systematic error $\Delta U(h)$, dispersion $\sigma_{\delta U^{\prime}}^{2}$ and $r m s$ deviations (variances) $\sigma_{\delta U}$ of the random component of errors with zero mean value, and inter-layer covariance $B_{\delta U}\left(h_{1}, h_{2}\right)$ and correlation functions $R_{\delta U}\left(h_{1}, h_{2}\right)$.

In Figure 1, altitude profiles of systematic and random errors parameters with the altitude distribution of ozone variations are given for total October and December ensembles of 1996-2017. 

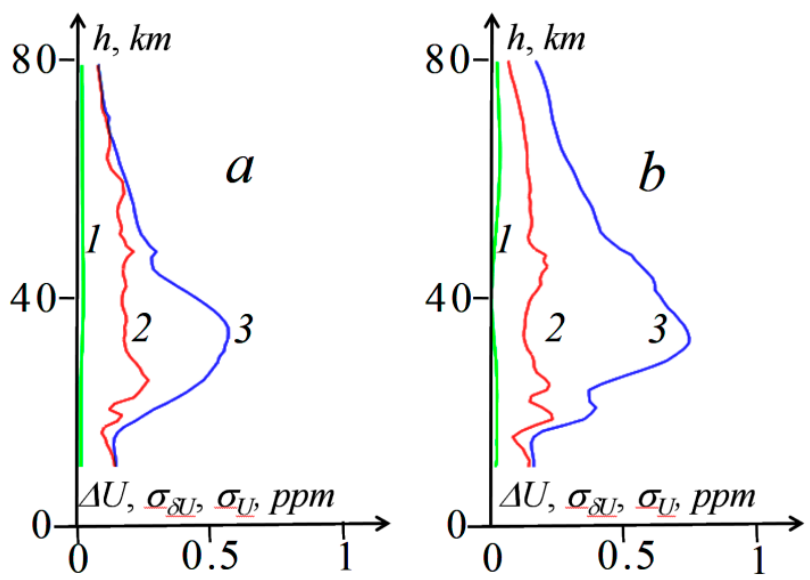

Figure 1. Profiles of ozone retrieval error parameters and ozone profile variations in 1996-2017: (a) October ensemble; (b) December ensemble. 1—systematic errors $\Delta U(h) ; 2$-rms random errors $\sigma_{\delta U}(h)$; 3-ozone $r m s$ deviations $\sigma_{U}(h)$ from the mean profile $<U(h)>$.

One can see that systematic errors are negligibly small; rms of random errors are very stable amounting to $0.1-0.2 \mathrm{ppm}$ in a wide altitude range and nearly independent on ozone variations and statistical ensemble. The measurements are informative approximately in the range from 20 up to $50-70 \mathrm{~km}$; their variances are seasonally dependent-it is possible to see that December variances are stronger and the informative region is wider than those in October.

The inter-level covariance $B_{\delta U}\left(h_{1}, h_{2}\right)$ and correlation $R_{\delta U}\left(h_{1}, h_{2}\right)$ functions of retrieval errors for the December ensemble are shown in Figure 2.
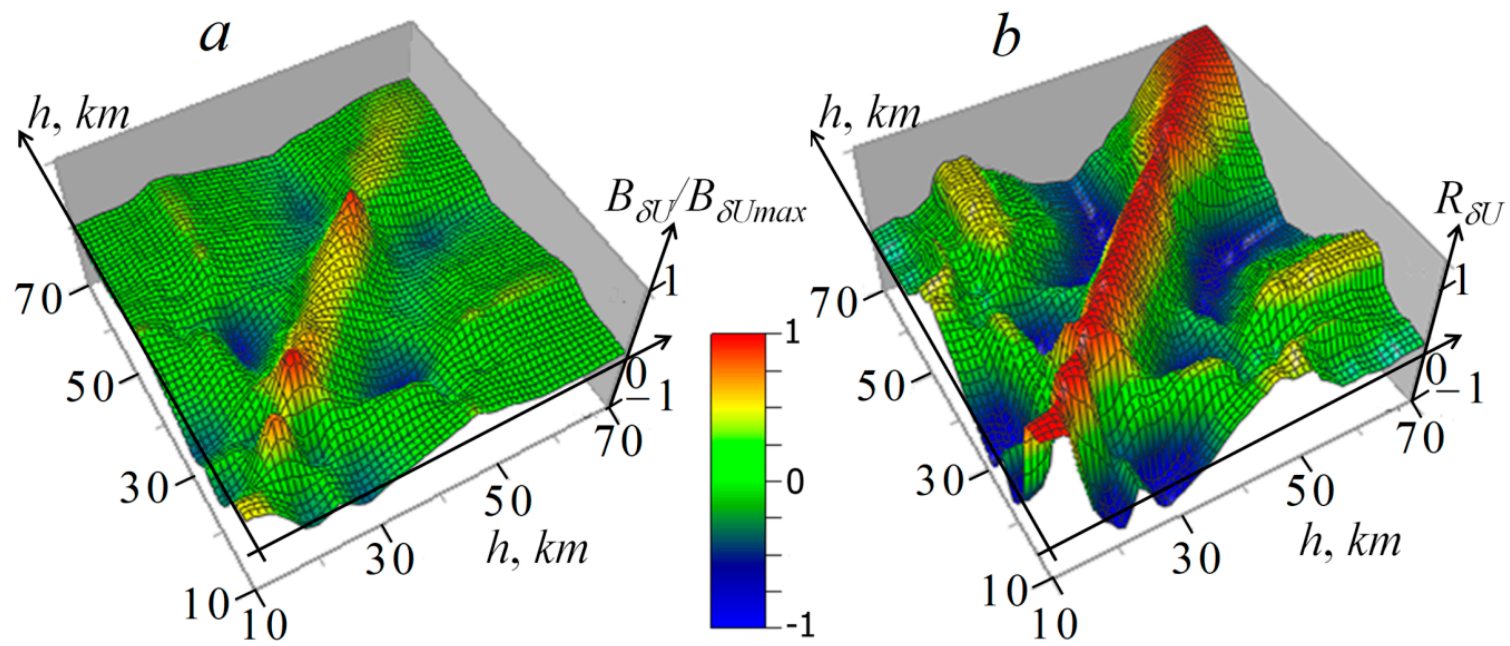

Figure 2. Inter-level covariance (a) and correlation (b) functions of retrieval errors for the December ensemble.

These functions (especially correlation functions) are rather similar for different month ensembles. It is seen that for altitudes $h>50 \mathrm{~km}$, the distribution of the correlation function became much wider and at $h>70 \mathrm{~km}$ errors appeared totally correlated. We think that it is related with the fading of the method sensitivity with altitude.

\subsection{Probability Distributions of Ozone Variations in Two Decades (1996-2006 and 2007-2017)}

In Figure 3, two decade ensembles of ozone profiles are demonstrated. 


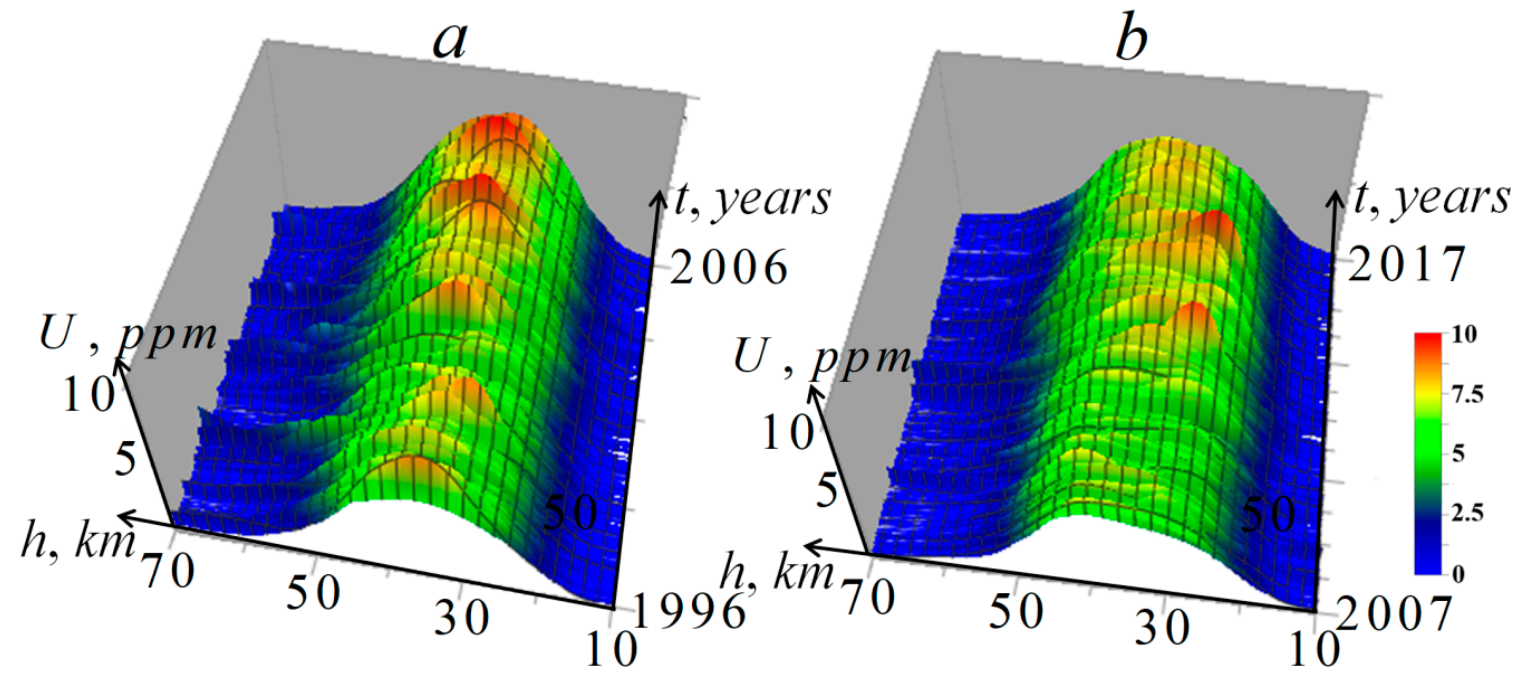

Figure 3. Retrieved day-time ozone profiles over Moscow. (a) ensemble of 1996-2006 profiles; (b) ensemble of 2007-2017 profiles.

It is possible to see, in Figure 3, strong variations of ozone profiles in the stratosphere over Moscow that include random and seasonal components. One can mention that profiles for the 2007-2017 decade (Figure 3b) look in some places somewhat broadened and decreased at altitudes of their maxima.

In Figure 4, one can compare distributions of the probability density of variations (deviations from the mean profiles $U(h)-<U(h)>$ ) calculated for these two ensembles.
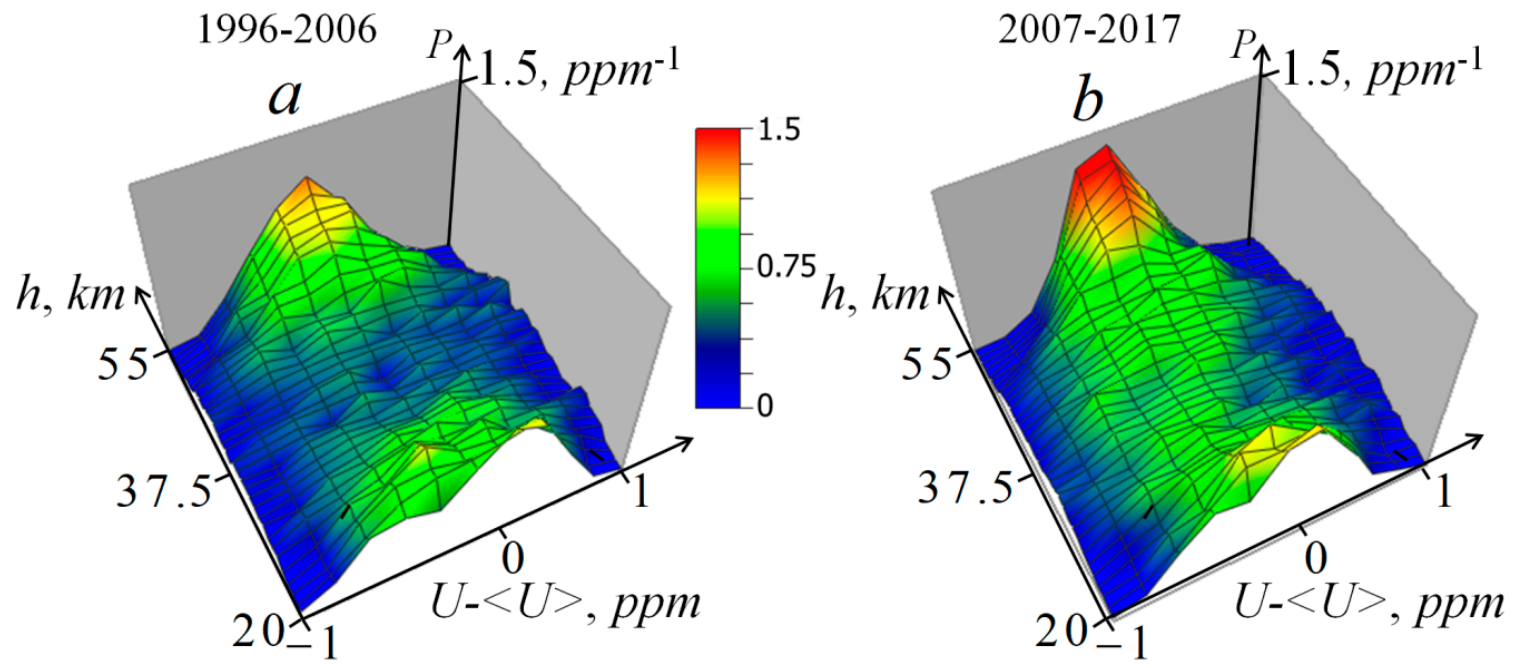

Figure 4. Distributions of probability density of ozone profile variations. (a) Ensemble of 1996-2006 profiles; (b) ensemble of 2007-2017 profiles.

As it is possible to see, in Figure 4, that probability distributions mostly have Gaussian-like shapes, and the probability density is much more widely distributed at altitudes of ozone profile maxima and more localized at lower and higher altitude levels. Additionally, there is a significant difference between results for these two ensembles-variations of 1996-2006 years are more widely distributed than those of 2007-2017, which leads to the significant decrease in mean-square variations in the 2007-2017 decade when compared to those in the decade 1996-2006. Based on the following statistical analysis, seasonal and decade changes of middle-latitude stratospheric ozone will be revealed more clearly.

Below, we demonstrate long-term changes in other statistical parameters, where, taking into account ozone seasonal variations, we began with statistical analysis for separate months. For this, 
nine separate sub-ensembles for every month of autumn, winter, and spring seasons were extracted from these two decade ensembles.

\subsection{Mean and Variances of Retrieved Ozone Profiles in Separate Months of Two Decades (1996-2006 and} 2007-2017)

In Figure 5, seasonal dynamics of the monthly mean ozone profiles $<U_{0}(h)>=\frac{1}{M} \sum_{i=1}^{M} U_{0 i}$ for months from September to May for the 1996-2006 and 2007-2017 decades are given ( $M$ is the number of ozone profiles in the corresponding month ensemble that includes profiles obtained in all days of measurements in this month in all eleven years of each of the two decades).

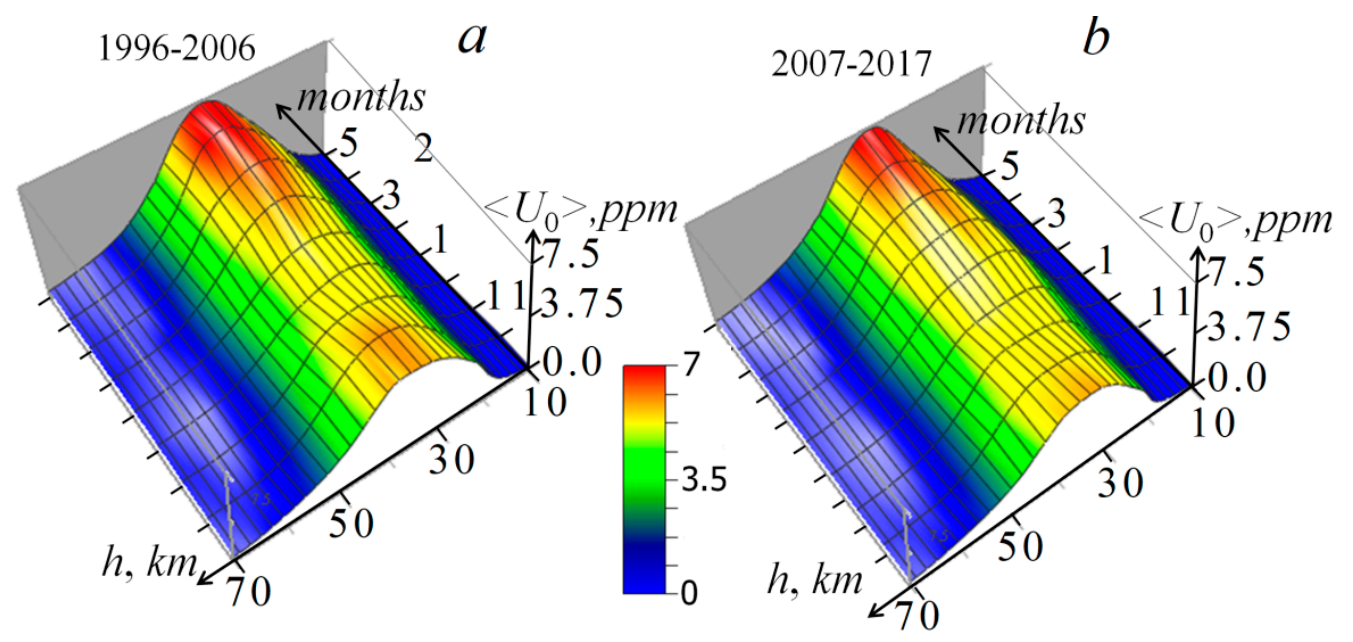

Figure 5. Seasonal variations of monthly mean ozone profiles averaged over decades 1996-2006 and 2007-2017 arranged from September (9th month) to May (5th month). (a) Ensemble of 1996-2006; (b) ensemble of 2007-2017.

The results shown in Figure 5 demonstrate noticeable seasonal variations of mean ozone profiles that are quite similar for both decades; however, it is possible to discern a decrease in maxima and a wider altitude distribution in cold months (from November to March) of the 2007-2017 decade that are seen much more clearly than in Figure 3. In the decade 1996-2006, the ozone VMR achieved its maximum value of $7.37 \mathrm{ppm}$ at the altitude of $36 \mathrm{~km}$ in April; the minimum value (at the altitude of the maximum of the ozone profile) of $5.27 \mathrm{ppm}$ was observed in December at $34 \mathrm{~km}$-so, the maximum seasonal variation amounted to $2.1 \mathrm{ppm}$ (28.5\% of the maximum value). In 2007-2017, the same values were $7.29 \mathrm{ppm}$ at $34 \mathrm{~km}$ in May and $5.22 \mathrm{ppm}$ at $34 \mathrm{~km}$ in December with almost the same difference of about $2.1 \mathrm{ppm}(28.4 \%)$.

In Figure 6, seasonal dynamics of monthly mean $r m s$ variances of retrieved ozone profiles averaged over eleven years of the 1996-2006 and 2007-2017 decades $\sigma=\sqrt{\frac{1}{M-1} \sum_{i=1}^{M}\left(U_{i}-<U>\right)^{2}}$ (where $M$ is the same as defined for monthly means in Figure 5) and variances of ozone profiles $\sigma_{0}$ cleared from retrieval errors are demonstrated. 

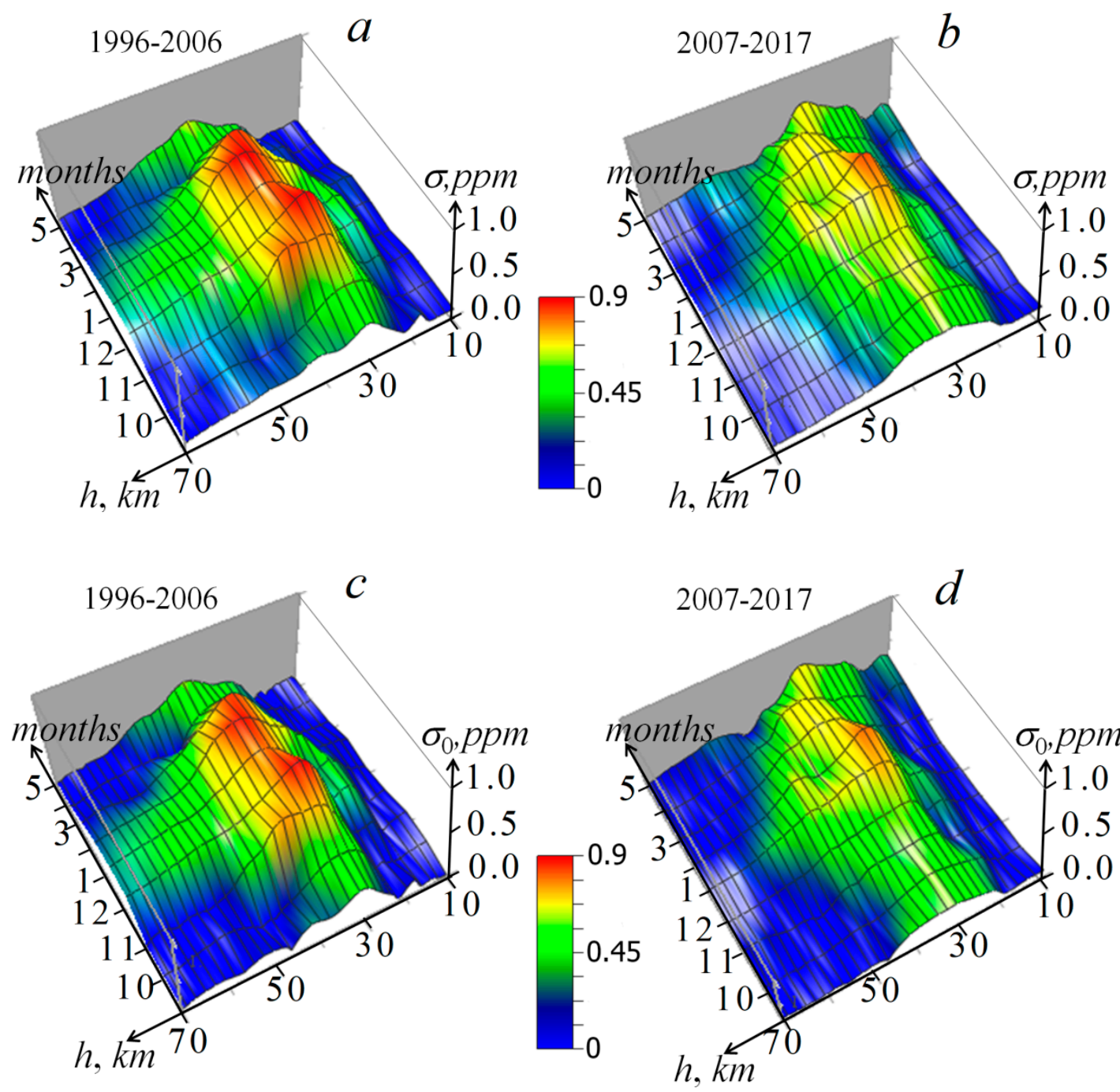

Figure 6. Seasonal changes in monthly mean rms variances of the ozone profiles averaged over decades 1996-2006 and 2007-2017. Upper row: variances obtained from ensembles of retrieved profiles; bottom row: variances cleared from retrieval errors. Results are arranged from September (9th month) to May (5th month). (a,c) Ensembles of 1996-2007; (b,d) ensembles of 2007-2017.

As clearly seen in Figure 6, in the distributions of both retrieved $(a, b)$ and cleared from error (c, d) parameters, ozone variances in the second decade (2007-2017) are essentially decreased as compared to the decade 1996-2006, especially in cold months, which confirms the results shown in Figure 4. It is also interesting to mention two maxima of variability at altitudes higher and lower than corresponding maxima of the ozone profile in the distribution of variances in the winter months of the decade 2007-2017. The maximal value of variances $\sigma_{0}$ in the decade 1996-2006 amounts to $0.92 \mathrm{ppm}$ at $32 \mathrm{~km}$ in December; in the decade 2007-2017, it was $0.83 \mathrm{ppm}$ at $30 \mathrm{~km}$ in February-both about $12 \%$ of the corresponding maximal mean values.

In Figure 7, one can see differences $\Delta<U_{0}>=\left\langle U_{0}>_{2007-2017}-<U_{0}>_{1996-2006}\right.$ and $\Delta \sigma_{0}=\sigma_{0}$ 2007-2017 $-\sigma_{0}$ 1996-2006 - between ozone parameters shown in Figures 5 and 6 and the distribution of $r m s$ sampling errors $\delta\left(\Delta<U_{0}>\right)=\sqrt{\left(\sigma_{\delta U 1996-2006}^{2}+\sigma_{\delta U 2007-2017}^{2}\right.}$. Sampling errors of covariance matrixes $B$ and $B_{2}$ are independent, so their effect is included at the correction of $r m s$ variances demonstrated in Figure 6, and appeared to be not significant. 


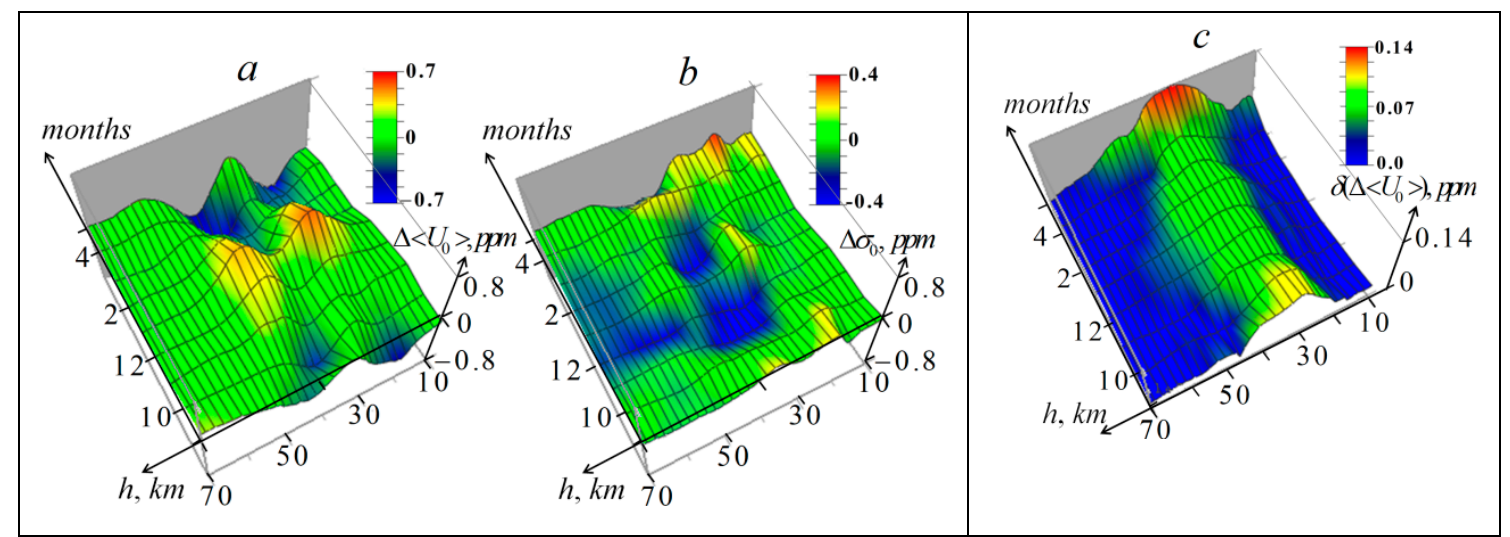

Figure 7. Differences in monthly mean ozone profiles and their variances between two decades shown in Figures 5 and 6: (a) difference between monthly mean ozone profiles; (b) difference between monthly mean rms ozone variances; (c) rms sampling errors of the difference shown in Figure 7a. Results are arranged from September (9th month) to May (5th month).

Relatively stronger decade changes of the monthly mean ozone content were revealed at altitudes higher and lower than the layer maxima positions in Figure $7 \mathrm{a}$. The maximal positive changes $(0.59 \mathrm{ppm}$ at $27 \mathrm{~km}$ and $0.52 \mathrm{ppm}$ at $44 \mathrm{~km}$ - both $12.6 \%$ of the mean values in Figure 5a) took place in January, whereas maximal negative changes were registered in September $(-0.47 \mathrm{ppm}$ at $19 \mathrm{~km}-32 \%)$, October $(-0.39 \mathrm{ppm}$ at $37 \mathrm{~km}-6.2 \%)$, and May $(-0.45 \mathrm{ppm}$ at $42 \mathrm{~km}-8.4 \%$ and $-0.50 \mathrm{ppm}$ at $19-22 \mathrm{~km}-26-18 \%)$. Corresponding sampling errors shown in Figure $7 \mathrm{c}$ are small enough to demonstrate the significance of the revealed changes. It is also seen that these errors are relatively small for cold months (because of the larger number of profiles in ensembles), and corresponding parameters are yet more statistically significant. It is also interesting to mention that the two-maximum structure of changes in January (Figure 7a) on the whole remain in other months, gradually changing in amplitude and shifting to different altitudes in both directions from this month.

In Figure 7b, we see that variability of the ozone layer over Moscow in the 2007-2017 decade was mostly decreased in comparison with 2006-2016, especially in cold months. Maximal strong decreases in the rms variances were detected at altitudes near the ozone profile maximum $(-0.35 \mathrm{ppm}$ at $33 \mathrm{~km}$ in December and at $39 \mathrm{~km}$ in February-decreases of 38 and $46 \%$ of the monthly mean variances in 1996-2006, respectively). The revealed noticeable decrease in the ozone layer variability in the middle stratosphere can be considered as the most important and unexpected result of this analysis.

For a better understanding, in Figure 8, relative differences $\Delta<U_{0}>, \%=$ $100\left(<U_{0}>_{2007-2017}-<U_{0}>_{1996-2006}\right) /<U_{0}>_{1996-2006,} \Delta \sigma_{0}, \%=100\left(\sigma_{0}\right.$ 2007-2017 $\left.\sigma_{0} 1996-2006\right) /<U>_{1996-2006}$, and distribution of relative $r m s$ sampling errors $\delta\left(\Delta<U_{0}>\right), \%=$ $100 \sqrt{\left(\sigma_{\delta U 1996-2006}^{2}+\sigma_{\delta U 2007-2017}^{2}\right.} /<U_{0}>_{1996-2006}$ are given only for the cold months (from November to March) and in a narrower range of altitudes around the ozone maximum.

In Figure 8, one can see that the general shape of relative changes in monthly mean ozone profiles (Figure 8a) and variances (Figure $8 \mathrm{~b}$ ) is similar: they have two maxima at altitudes higher and lower than the ozone layer maximum (most noticeable in January) and minima at altitudes of the ozone layer maximum. Decade changes in the variances within the region shown in Figure 8 are comparable to changes in the mean profiles: changes of variances span the interval from -7 to $3 \%$ (mainly in the negative region), whereas the mean profiles change rather symmetrically from -9 to $14 \%$. The level of sampling errors demonstrated in Figure $8 \mathrm{c}$ spans values from 0.8 to $1.9 \%$ that confirm statistical significance of the revealed unexpectedly strong offsets. 


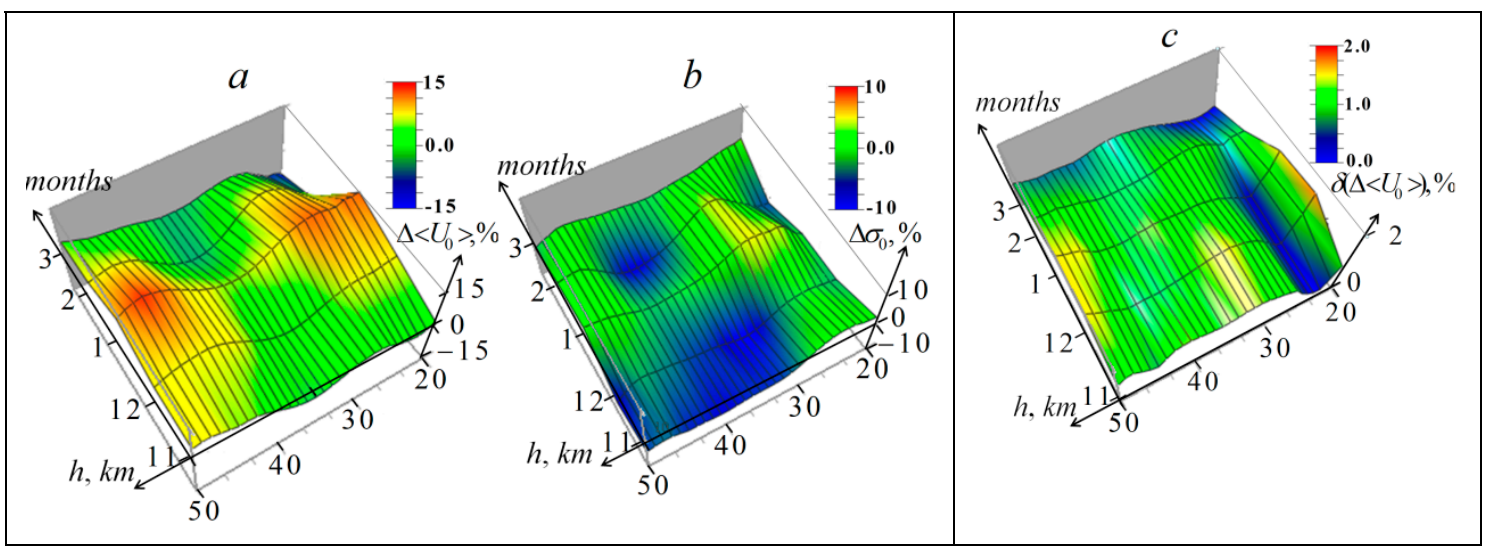

Figure 8. Differences in relative monthly mean ozone profiles and their rms variances between the two decades, in percent: (a) difference between monthly mean ozone profiles; (b) difference between monthly mean rms ozone variances; (c) rms sampling errors of mean ozone profiles difference. The results are arranged from November (11th month) to March (3th month).

Below, we demonstrate seasonal features and changes of correlation parameters for each month averaged over years of each of the two decades.

\subsection{Inter-altitude and Time Covariance and Correlation Functions in Separate Months of Two Decades} (1996-2006 and 2007-2017); Frequency Spectra of Time Covariance and Correlation Functions

In Figure 9, inter-altitude covariance functions cleared from retrieval errors are demonstrated.

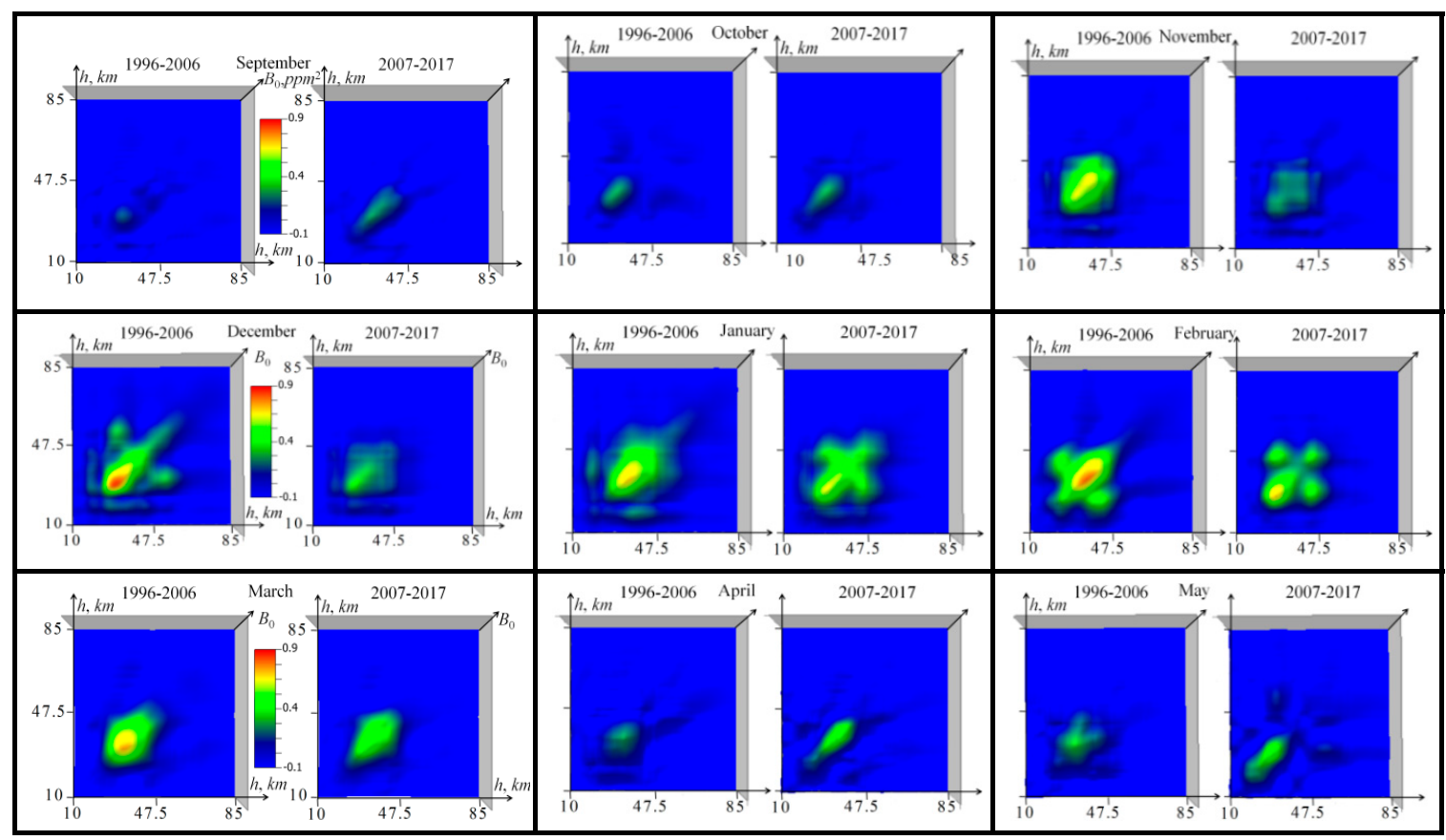

Figure 9. Inter-altitude covariance functions for autumn (top row), winter (middle row), and spring (lower row) seasons calculated for decades 1996-2006 and 2007-2017.

Results shown in Figure 9 demonstrate a large seasonal diversity and significant decade changes of inter-level covariance relations that can be interesting for studies of the atmosphere long-term dynamics. The variability of the ozone layer was considerably decreased in the cold months (from November to March) of the decade of 2007-2017 if compared to that in the 1996-2006 decade, whereas it remained unchanged or somewhat increased in near-summer months. 
Corresponding inter-altitude correlation features are demonstrated in Figure 10, where noncleared correlation functions $R$ for retrieved ozone profiles are shown (because the cleared correlation functions $R_{0}$ have strong distortions in regions of small variances).

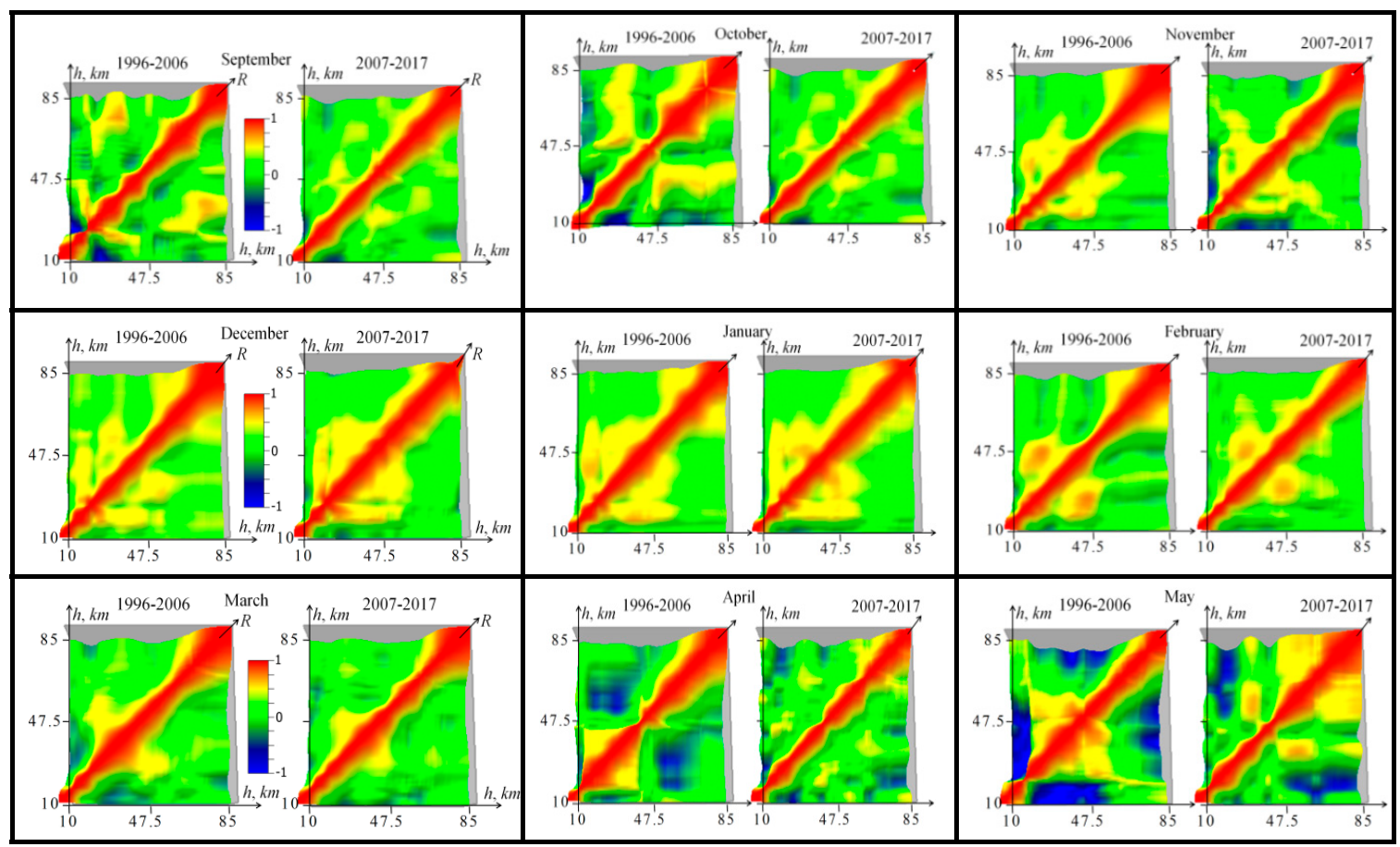

Figure 10. Inter-altitude correlation functions for autumn (top row), winter (middle row), and spring (lower row) seasons calculated for decades 1996-2006 and 2007-2017.

One can see here a seasonal diversity and decade changes of inter-level relations, though, on the whole, inter-altitude correlation is unexpectedly stable. Additionally, in most cases, it is worth mentioning that the broadening of high-correlation ranges above altitudes $50-60 \mathrm{~km}$ is related to a decrease in sensitivity and resolution of the microwave method with altitude.

In Figure 11, the time covariance functions are given depending on the time shift $\tau$, where more strong seasonal diversity and decade changes are seen.

The results also demonstrate that the seasonal ozone variability is decreased in cold months of the decade 2007-2017 when in comparison with that in the 1996-2006 decade, whereas it increased in near-summer months. Furthermore, in these months, secondary maxima are observed that can be related to some periodical nonstationarity. It is important to mention that random errors of various origin (including those related to errors in the temperature profile) are independent at $\tau>0$, and, hence, they are efficiently suppressed when averaging, whereas systematic errors are eliminated in time covariance functions. This leads to the much higher statistical significance of these statistical parameters. 


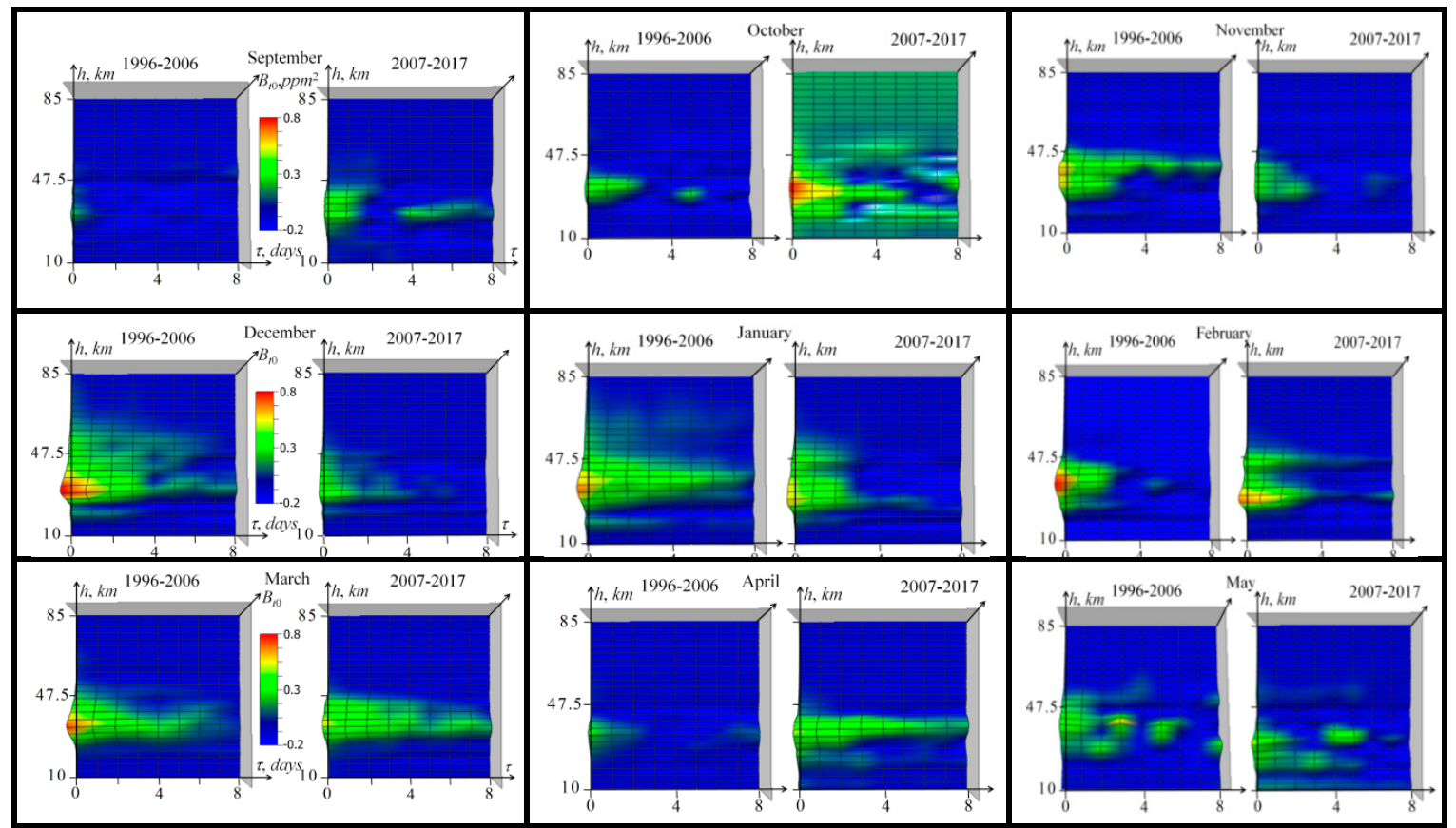

Figure 11. Time covariance functions for autumn (top row), winter (middle row), and spring (lower row) seasons calculated for decades 1996-2006 and 2007-2017.

The seasonal and decade changes presented in Figure 11 manifested themselves in frequency spectra of time covariance functions given in Figure 12 for the frequency band from 0 up to 0.5 days $^{-1}$ (the Niquist frequency).

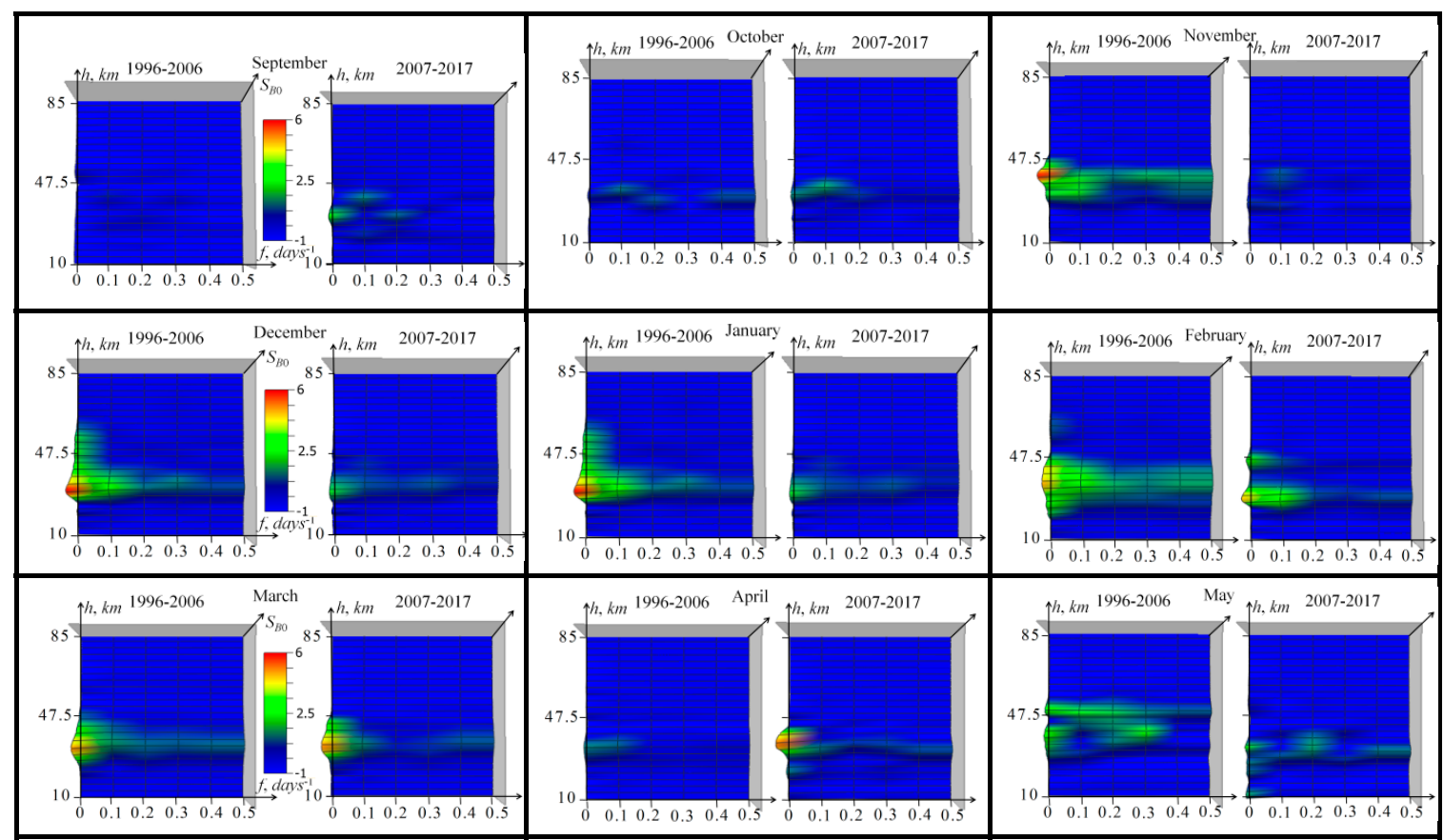

Figure 12. Spectrum of covariance functions for autumn (top row), winter (middle row), and spring (lower row) seasons calculated for decades 1996-2006 and 2007-2017.

It is seen that spectra for cold moths represent the decrease in the ozone profile variability in the 2007-2017 decade. Secondary maxima observed in some months can be related to periodic processes. 
In Figure 13, corresponding time correlation (autocorrelation) functions are demonstrated.

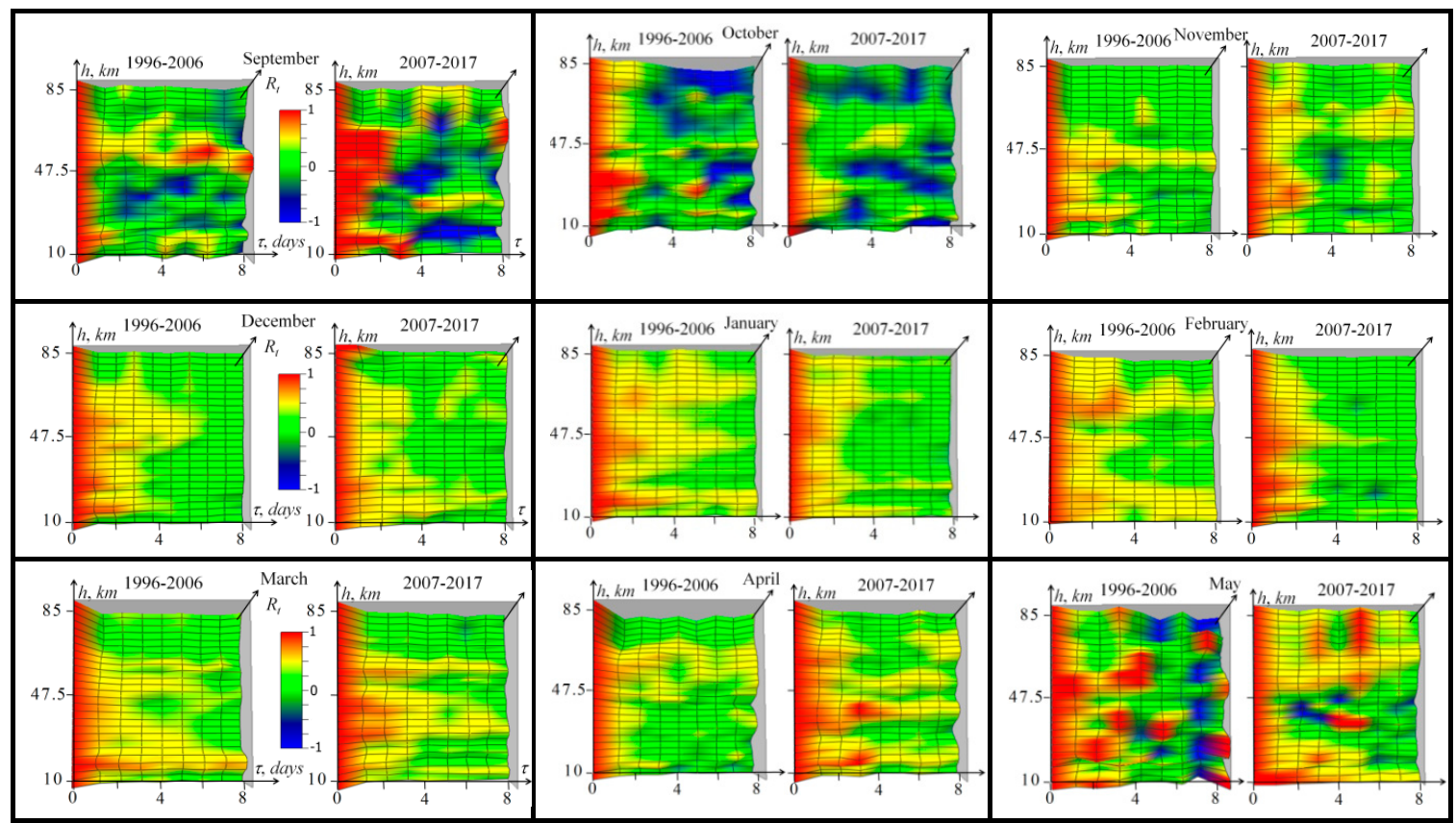

Figure 13. Time correlation (autocorrelation) functions for autumn (top row), winter (middle row), and spring (lower row) seasons calculated for decades 1996-2006 and 2007-2017.

In Figure 13, it is possible to see that distributions of correlation functions sharply decrease with time shift and are very different for cold and near-summer months. For cold months, they monotonously decrease for both decades, but it is seen that for the decade 2007-2017, the correlation time mainly increases, especially in some altitude ranges, which is a clear manifestation of the decrease in the ozone variability in corresponding layers. For summer months, a strong diversity is seen, and the time correlations are quite different from cold months: there are multiple second maxima and minima with a negative correlation that can result from nonstationarity related to large-scale dynamical processes in the stratosphere and propagation of planetary waves or other periodical processes. The typical time of ozone correlation function in Figure 13 is 1-3 days; however, in the above-mentioned cases, more long-term correlations remain of up to 8 days and more.

The frequency spectra of the correlation functions in Figure 13 are given in Figure 14.

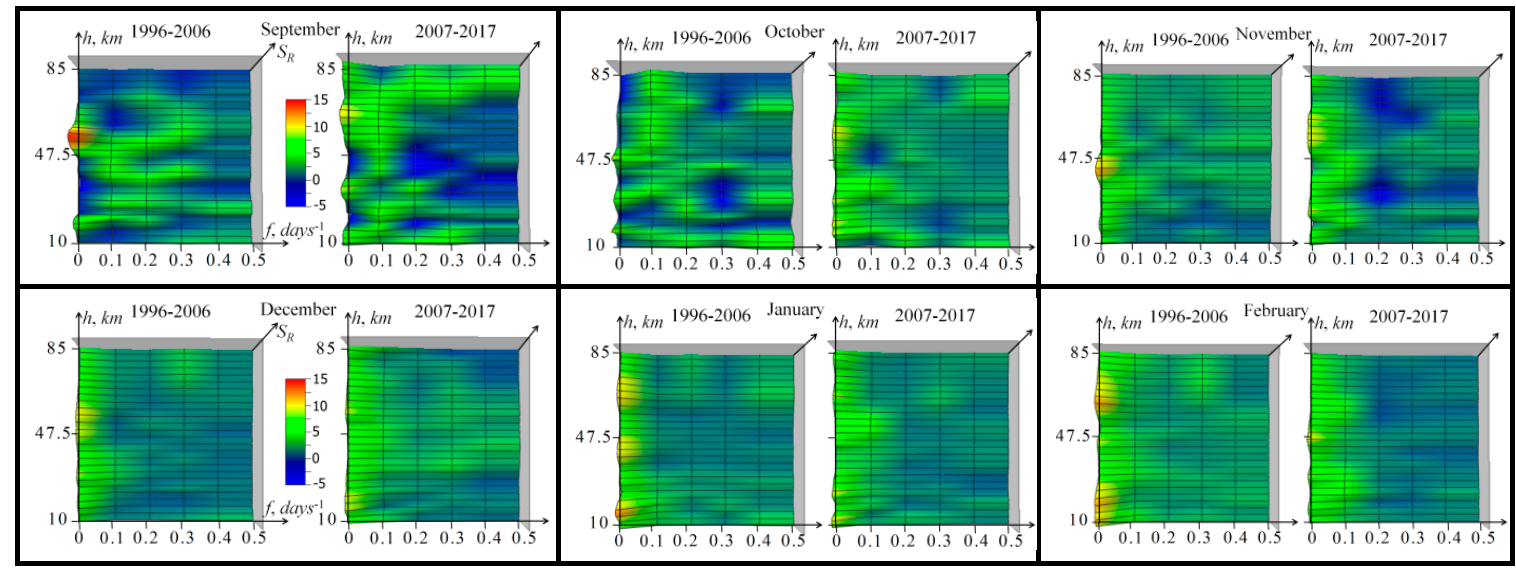

Figure 14. Cont. 


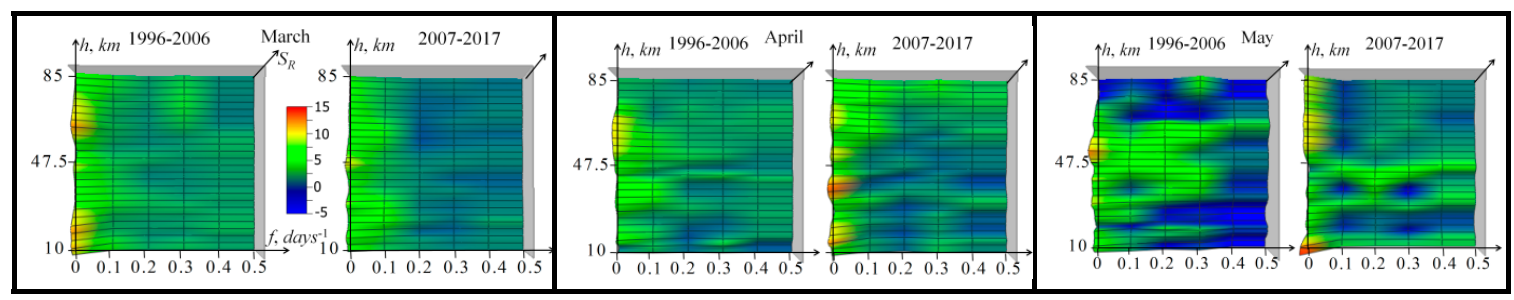

Figure 14. Spectrum of correlation functions for autumn (top row), winter (middle row), and spring (lower row) seasons calculated for decades 1996-2006 and 2007-2017.

\subsection{Integral Statistical Parameters of Ozone Profiles of Two Decades (1996-2006 and 2007-2017).}

It is also interesting to average the monthly mean statistical parameters over all the months from September to May to compare seasonally-independent integral parameters for 1996-2006 and 2007-2017 decades. It is possible to reveal some general features of decade ozone offsets using averaged monthly mean (integral mean) parameters:

$$
<U_{0}^{\text {int }}(h)>=\frac{1}{M} \sum_{i=9}^{5}<U_{0 i}>, \sigma^{\text {int }}(h)=\sqrt{\frac{1}{M} \sum_{i=9}^{5} \sigma_{i}^{2}}, \sigma_{0}^{\text {int }}(h)=\sqrt{\frac{1}{M} \sum_{i=9}^{5} \sigma_{0 i}^{2}}, \sigma_{\delta U}^{\text {int }}(h)=\sqrt{\frac{1}{M} \sum_{i=9}^{5} \sigma_{\delta U i}^{2} \Delta<}
$$

$U_{0}^{\text {int }}(h)>=<U_{02007-2017}^{\text {int }}(h)>-<U_{01996-2006}^{\text {int }}(h)>, \Delta \sigma_{0}^{\text {int }}(h)=\sigma_{02007-2017}^{\text {int }}(h)-\sigma_{01996-2006}^{\text {int }}(h)$, $\Delta \sigma_{0}^{\text {int }}(h)=\sigma_{2007-2017}^{\text {int }}(h)-\sigma_{1996-2006}^{\text {int }}(h)$, and corresponding errors of the difference of integral mean

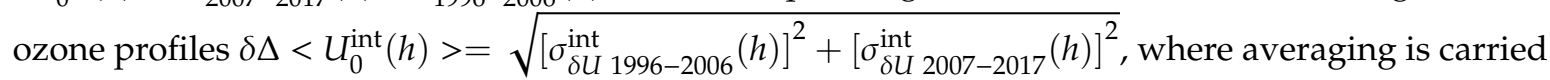
out over $\mathrm{M}=9$ months from September $(i=9)$ to May $(i=5) ; i$ is the month number.

In Figure 15a, averaged monthly mean ozone profiles and their averaged variances are shown.

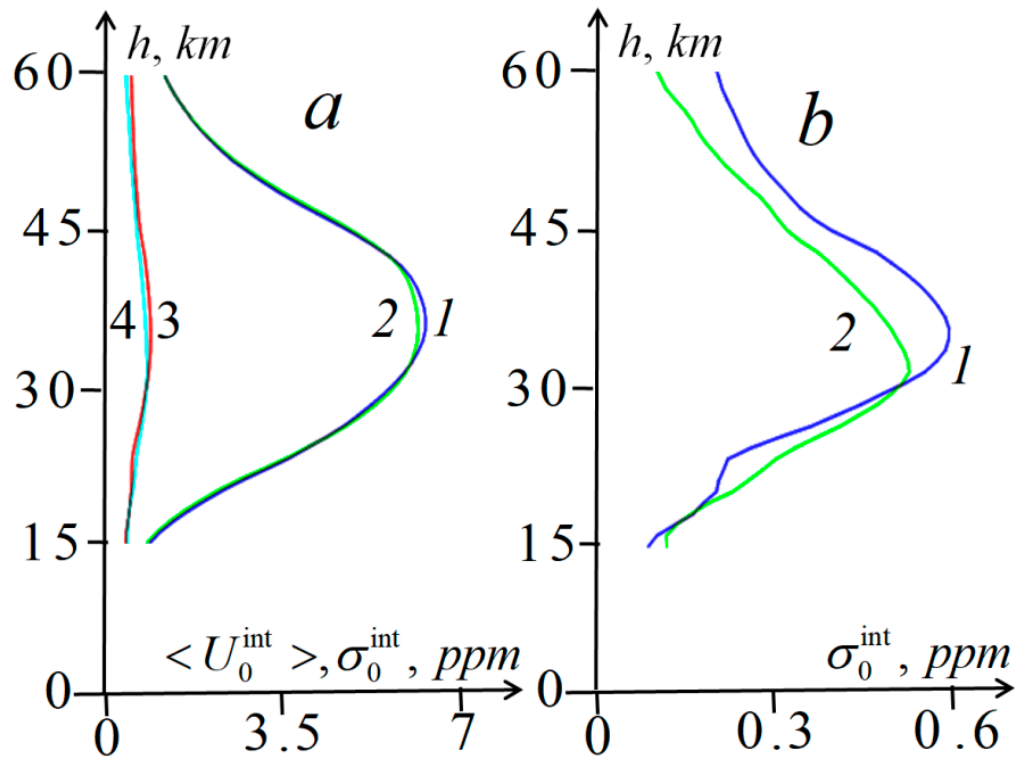

Figure 15. Profiles of ozone integral mean and rms variances: (a) 1,2-profiles of ozone integral mean $<U_{0}^{\text {int }}$ 1996-2006 $(h)>$ and $<U_{02007-2017}^{\text {int }}(h)>; 3$, 4-profiles of ozone rms variances $\sigma_{01996-2006}^{\text {int }}(h)$, $\sigma_{02007-2017}^{\text {int }}(h)$; (b) 1, 2-profiles of ozone rms variances $\sigma_{01996-2006}^{\text {int }}(h), \sigma_{02007-2017}^{\text {int }}(h)$ (the same as in Figure 15a).

One can see that strong differences in monthly mean profiles between two decades (see Figures 6 and 7) are considerably smoothed in the integral mean decade profiles so that only a small decrease in the maximum can be discerned clearly, whereas it is difficult to discern two maxima at higher and lower altitudes. The smoothing of the changes in the integral mean profile and variances averaged 
over nine months is related to the seasonal diversity in altitude distributions of monthly mean decade changes (Figure 7).

Changes of the integral ozone profile variances are clearly seen in Figure 15b; a decrease at $h>30 \mathrm{~km}$ and an increase at $h<30 \mathrm{~km}$ remain rather significant. Together with the revealed decade changes in monthly mean variances (see Figures 6 and 7) this should be considered as the most important result of this research.

In Figure 16, the decade differences between the integral parameters shown in Figure 15 are given.

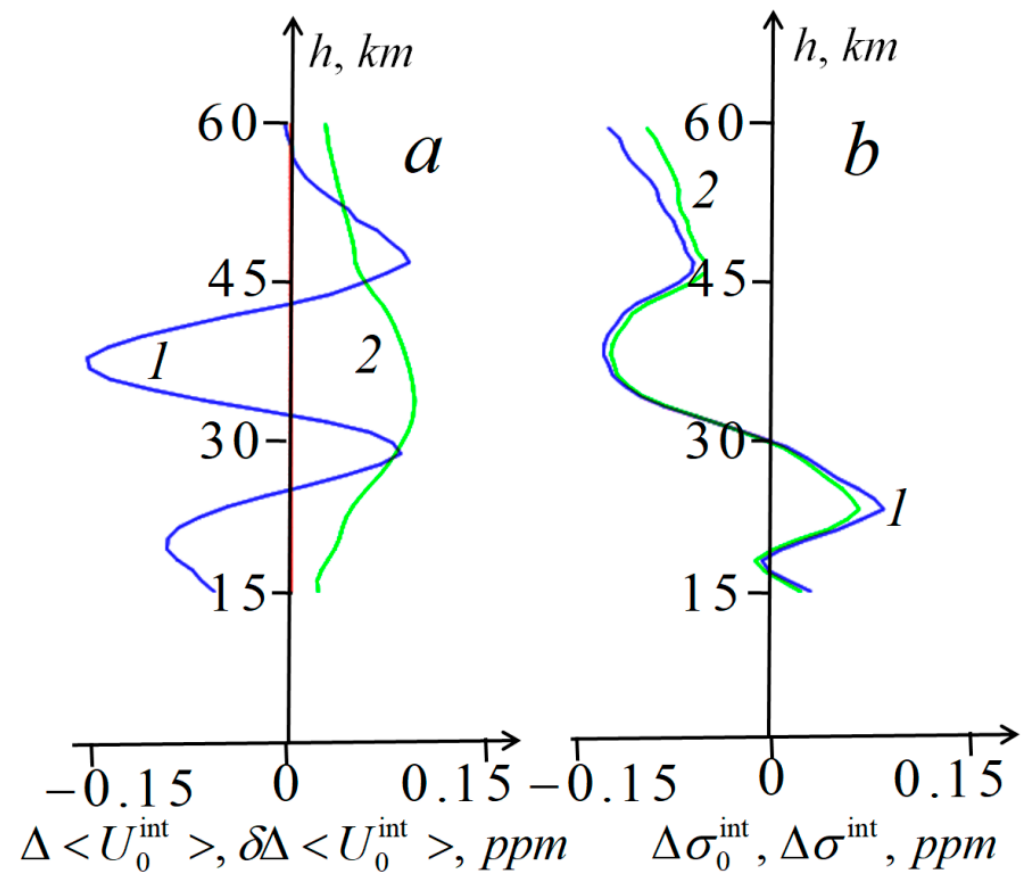

Figure 16. (a) 1 -altitude profile of the difference $\Delta<\mathrm{U}_{0}$ int $>$ between integral mean ozone profiles for ensembles of 2007-2017 and 1996-2006; 2-profile of corresponding rms sampling errors $\delta \Delta<\mathrm{U}_{0}{ }^{\text {int }}>$; (b) 1-profile of the difference $\Delta \sigma_{0}$ int between integral ozone variances for ensembles 2007-2017 and 1996-2006; 2-profile of the difference between corresponding noncleared variances $\Delta \sigma^{\text {int }}$.

In this figure, it is more clearly seen that strong changes in monthly mean profiles for separate months between two decades (see Figures 6 and 7) are considerably smoothed in integral mean ozone differences. Moreover, sampling errors of these changes are somewhat increased because they include the deposition of seasonal variations. The decade integral mean ozone changes have the altitude distribution similar to that in the monthly mean changes for winter months (see Figure 7a): decreases by $5.7 \%$ at the altitude of $19 \mathrm{~km}$ and by $2.6 \%$ at $37 \mathrm{~km}$ with sampling errors by $1.5 \%$; increases by $1.7 \%$ at $28 \mathrm{~km}$ and $2.5 \%$ at $47 \mathrm{~km}$ with errors of $1.6 \%$. The corresponding decade averaged mean column (integral) ozone content above $20 \mathrm{~km}$ remained practically unchanged: $4.61 \mathrm{~g} / \mathrm{m}^{2}$ for the decade $1996-2006$ versus $4.58 \mathrm{~g} / \mathrm{m}^{2}$ for $2007-2017$.

Changes of the integral mean variances shown in Figure 16b both for cleared and non-cleared variances are comparable to those of monthly mean variances (see Figure $7 \mathrm{~b}$ ) and demonstrate the deep decrease at altitudes above $30 \mathrm{~km}$ with the maximum at $38 \mathrm{~km}(-25 \%$ of the value of integral variances for 1996-2006 at this altitude, shown in Figure 15b) and an increase at altitudes below $30 \mathrm{~km}$ with the maximum at $23 \mathrm{~km}(35 \%)$. As seen in Figure 16b, these changes are quite similar for cleared and noncleared variances; here, it is noteworthy that absolute values of the latter offsets are somewhat less than those of the cleared one. Sampling errors in the cleared integral variances can be related only to errors of the clearing method, which are, in fact, unknown. However, they obviously cannot exceed the correction itself-i.e., they are for certain less than the difference between cleared and noncleared 
changes in Figure 16b. Even if one considers this difference as the standard deviation (sigma) of methodic errors, the significance of the revealed variances greatly exceeds the three-sigma criterion.

The similar changes of the ozonosphere were discovered for integral inter-altitude covariance functions. In Figure 17, the cleared covariance functions for two decade integral ensembles are shown together with their difference.

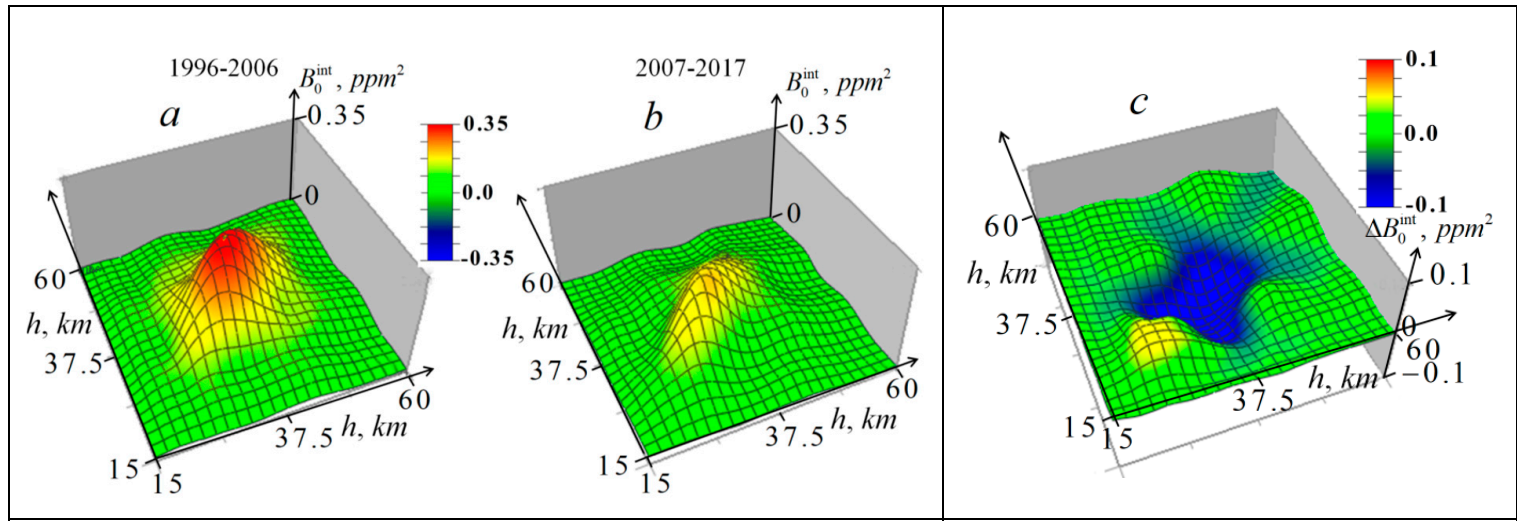

Figure 17. Inter-altitude integral mean covariance functions of ozone profiles: (a) ensemble of 1996-2006; (b) ensemble of 2007-2017; (c) difference $\Delta B_{0}^{\text {int }}(h)=B_{02007-2017}^{\text {int }}(h)-B_{01996-2006}^{\text {int }}(h)$

In Figure 17, one can see that the inter-altitude integral mean covariance functions also have drastic changes: the distribution of the covariance functions for 2007-2017 is strongly decreased at altitudes 30-45 km. In these functions for 2007-2017, one can also see secondary maxima. The corresponding regions of positive and strong negative changes are more clearly shown in Figure $17 \mathrm{c}$, where the difference of the covariance functions is provided.

In Figure 18, noncleared inter-altitude integral mean correlation functions for the decade ensembles are shown. It is seen that unlike the integral covariance function shown in Figure 17, integral correlation functions are practically unchanged: only a small decrease in correlation can be discerned at altitudes of the ozone layer maximum for the 2007-2017 ensemble.
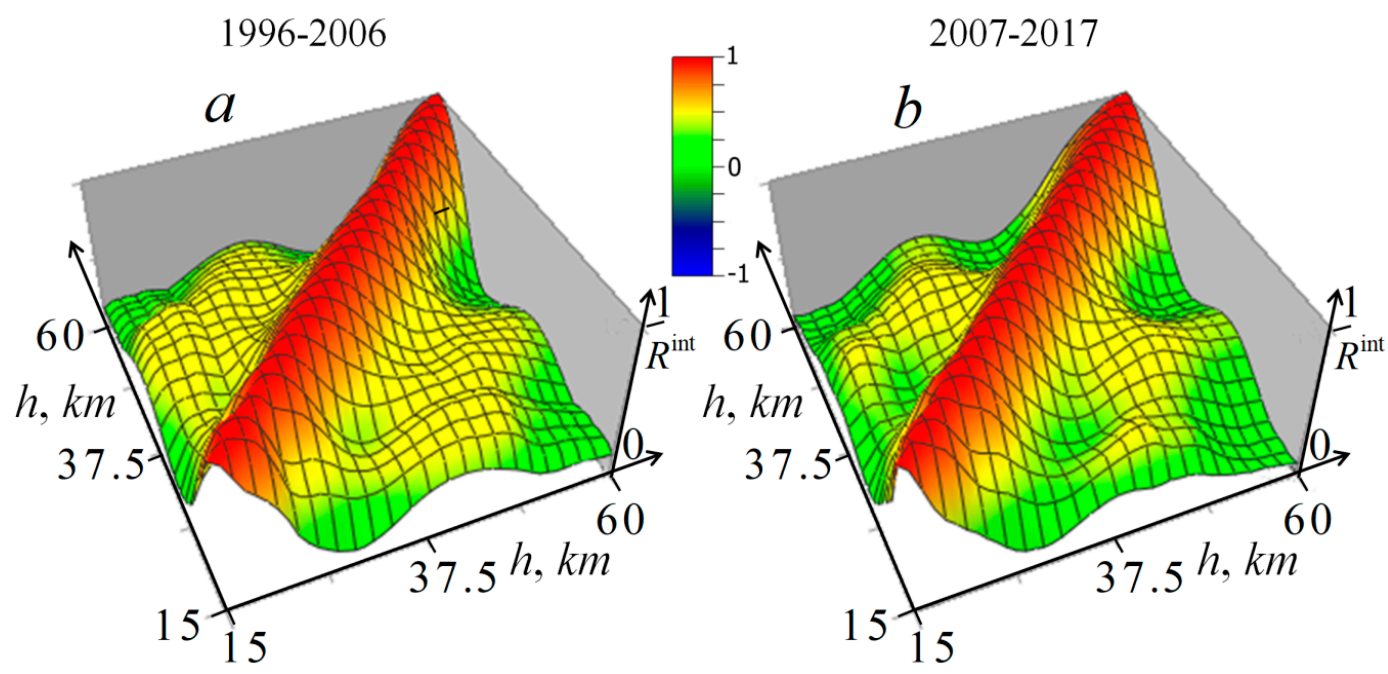

Figure 18. Inter-altitude integral mean correlation functions of ozone profiles: (a) ensemble of 1996-2006; (b) ensemble of 2007-2017.

It is noteworthy that several times in the decade 2007-2017, the stratospheric polar vortex was inclined so that the lower and middle stratosphere over Moscow was inside the vortex, while the upper stratosphere (40 km and higher) was outside the vortex. The cases resulted in different correlations of 
ozone concentration with physical parameters of the stratosphere (temperature, potential vorticity) and, correspondingly, in lower inter-level correlations. This could contribute to the small decrease in integral mean inter-level correlation for the decade 2007-2017 seen in Figure 18.

In Figure 19, integral mean time-covariance functions of ozone profiles cleared from the retrieval errors are given.
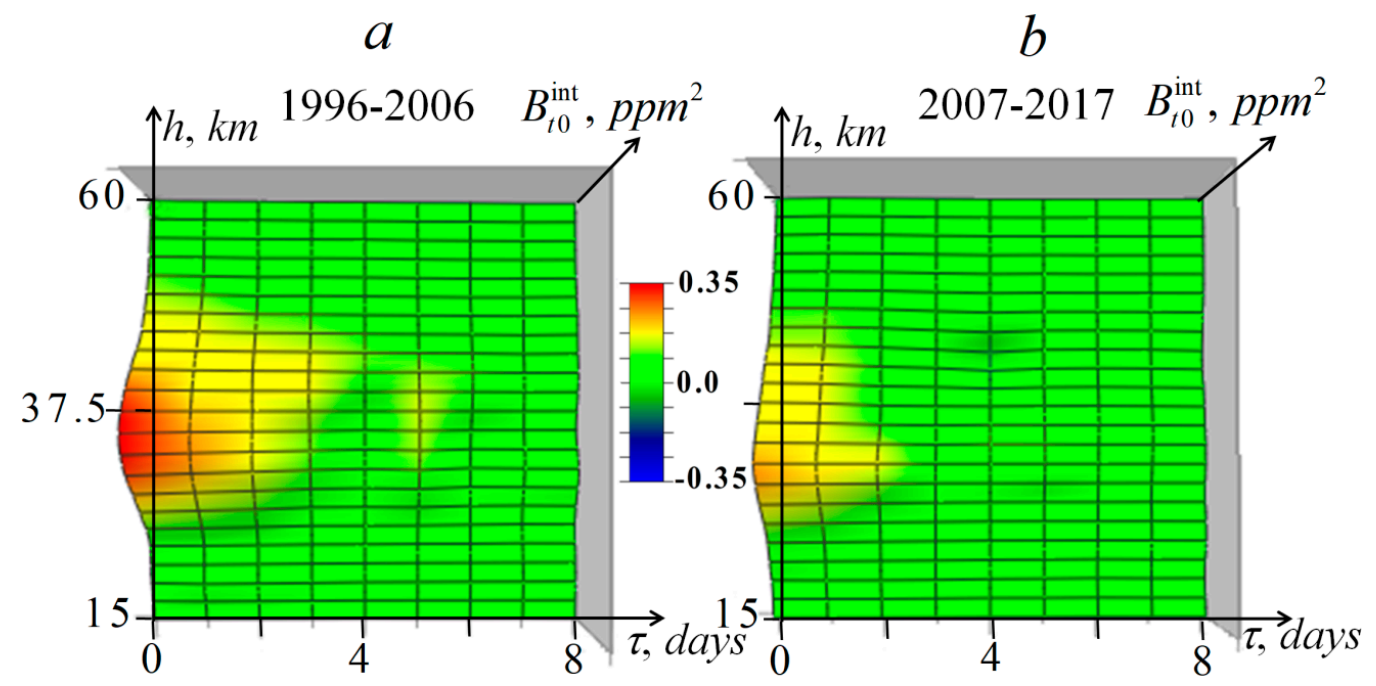

Figure 19. Cleared integral mean time-covariance functions of ozone profiles: (a) ensemble of 1996-2006;

(b) ensemble of 2007-2017.

Comparing results for the two decade ensembles, we can conclude that, like in the case of integral inter-altitude covariance function shown in Figure 17, there is a strong decade change revealed in the decrease in integral mean time-covariance functions at altitudes of $27-47 \mathrm{~km}$. Additionally, it is possible to discern small second maxima of the functions at $\tau=5$ days seen for both ensembles at altitudes near the ozone layer maximum.

Here, we stress again that the time-correlation parameters are obtained from much more independent (because of time shifts) data, and, hence, they are more reliable and statistically significant. Furthermore, because of this reason, it appeared possible to avoid strong distortions in the cleared time-correlation functions presented in Figure 20.
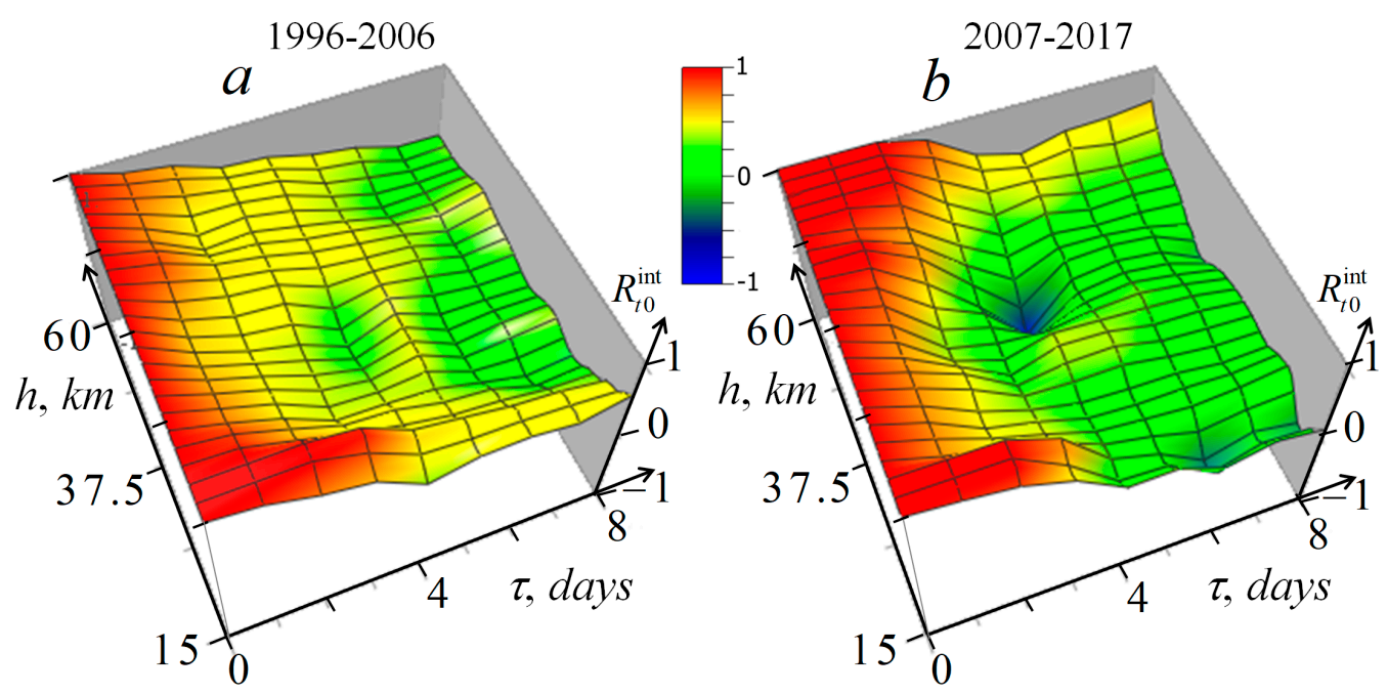

Figure 20. Cleared integral mean time-correlation functions of ozone profiles: (a) ensemble of 1996-2006; (b) ensemble of 2007-2017. 
It is seen in Figure 20 that the inter-altitude correlation functions for the decade 1996-2006, like the corresponding monthly mean correlation functions in Figure 13, have a shorter typical correlation time than those for the 2007-2017 decade, which means a decrease in the variability. Additionally, it is possible to the see the second maximum of functions at $\tau=5$ days at altitudes near the ozone layer maximum with the deep negative minimum at $\tau=4$ days that can be related to some long-remained nonstationarity.

\subsection{Comparison of Results with Other Research}

In the last two decades, many long-term measurements of composition and physical parameters of the middle atmosphere have been carried out using both ground-based and satellite-borne instruments. Among them, results of our research are especially important because its beginning in 1996 practically coincided with the beginning of the ozone recovery since 1997 reported in the most recent and comprehensive review [24]. As noted in [24], since 1997, in the middle and high latitudes, the increase in the total ozone column was small (about $1 \%$ per decade) with large inter-annual variations of about $5 \%$. Results of our 22-year study show that the decade offset of integral mean ozone content above $20 \mathrm{~km}$ was insignificant and negative-about $-0.7 \%$.

As for changes in the ozone profile, its trend in 1997-2016 for latitude zone 35-60N was estimated in [24] as $2-3 \%$ at a pressure level of $2 \mathrm{mbar}(42 \mathrm{~km}), 1 \%$ at $10 \mathrm{mbar}(31 \mathrm{~km})$, and $-2-0 \%$ at $70 \mathrm{mbar}$ $(19 \mathrm{~km})$. So, the trend was positive in the upper stratosphere and decreased to zero and small negative values in the lower stratosphere (see Figure 3.21 in [24]).

Since 2005, numerous comparisons of the LPI ozone profiles with profiles obtained by the MLS Aura instrument [25] over Moscow were performed [26,27]. The comparisons showed that in most cases, the LPI profiles coincided with the MLS ones within the experimental errors of the instruments for both the quiet state of the stratosphere and disturbed cases when ozone profiles with two maxima were observed.

The closest to Moscow ground-based mid-altitude station with a long-time set of ozone profiles is Bern $\left(46.57^{\circ} \mathrm{N}, 7.26^{\circ} \mathrm{E}\right)$ where regular ozone measurements with the GROMOS microwave radiometer operated at the same frequency of $142.2 \mathrm{GHz}$ were carried out in 1997-2015 [28]. A comparison of data presented in Figure 3 of the paper with analogous data from [28] showed that seasonal variations in Bern were less pronounced than in Moscow. Changes in integral mean ozone profiles obtained in our research (see Figures 15 and 16) correspond qualitatively to the trends [28] in the upper and lower stratosphere but are quite different (sign-changing) in the middle stratosphere at altitudes of $25-40 \mathrm{~km}$. Here, it should be noted that our data covered only nine months of three seasons (from September to May). Furthermore, they are local, and it is known that results are dependent on the geographical position of the observation points, especially in the north part of middle latitudes. For example, at the same latitude $55 \mathrm{~N}$, a shift of the stratospheric polar vortex from the North Pole to Europe in cold months produces quite different (opposite) effects on the stratospheric ozone over Moscow and the Pacific Ocean. As a result, local data on the ozone in the stratosphere for cold months may differ from the zonal mean estimates presented in [24]. Probably the main reason for the difference is the lower latitude of Bern ( $47 \mathrm{~N}$ versus $56 \mathrm{~N}$ for Moscow), so not all shifts of the stratospheric polar vortex to Europe in cold months resulted in noticeable ozone decreases, and the vortex edge rarely passed over Bern.

The decade trends for 1997-2015 revealed from the GROMOS data were $3.14 \pm 1.71 \%$ at $37 \mathrm{~km}$ and $-3.94 \pm 2.73 \%$ at $59 \mathrm{~km}$ [28]. On the whole, the trend obtained in Bern more or less corresponds to [24] altitudes lower than $45 \mathrm{~km}$, but is quite different from [24] those above this altitude. Our results at these altitudes give $-2.6 \pm 1.5 \%$ at $37 \mathrm{~km}$ and $-0.5 \% \pm 3.1 \%$ at $59 \mathrm{~km}$. The obtained altitude distributions of decade changes are both alternating-sign but rather different (compare Figure 16 in this paper with Figure 9 in [28]). The World Meteorological Organization ([29], Table 2.4) reported the decade ozone increase of $3.9 \pm 1.3$ at $40 \mathrm{~km}$ in the upper stratosphere at northern midlatitudes $\left(35-60^{\circ} \mathrm{N}\right)$ over the 2000-2013 period. 


\section{Discussion}

The results presented in this paper were rather unexpected. The large dimensions of statistical ensembles of the ozone profiles extracted from data which were obtained for the nine months from September to May for each of the two decades 1996-2006 and 2007-1017, and the applied method of clearing of the data from methodical errors provided a high enough statistical significance for our main results. The study of monthly mean ozone profiles, their $r m s$ variances, inter-altitude covariance and correlation functions, time covariance and correlation functions, and their frequency spectra revealed seasonal and decade changes of these parameters, especially the strong decrease in ozonosphere variability in cold months of 2007-2017 at altitudes above $30 \mathrm{~km}$. Furthermore, we averaged these parameters over all the months. The decade changes of such integral mean ozone profiles appeared smoothed and mostly not sufficiently statistically significant, whereas the averaged parameters of their variances demonstrated the strong decrease in ozonosphere variability above $30 \mathrm{~km}$ in the decade 2007-2017.

Our method of the retrieval errors correction based on their statistical analysis in the numerical simulation with ensembles of retrieved profiles enabled us to obtain more reliable results. However, it should be mentioned that it appeared difficult to take into consideration some errors related to calibration, apparatus function, as well as the influence of temperature profile errors on the kernel of the solved integral equation. One can expect that the effect of these sources of the retrieval errors can be compared to that of the signal noise. If so, the corresponding unaccounted correction of the covariance functions can be comparable to that of noise that were taken into account in the results demonstrated in Figures 6 and 16-i.e., rather inessential—especially because some of these unaccounted corrections should be correlated for different days. Consequently, the main results will be practically unchanged-especially bearing in mind that there is no reason to expect some seasonal and decade offsets of these errors. Hence, they could not significantly change the main results. In this paper, time-correlation functions were calculated for monthly mean ozone profiles and for integral mean profiles averaged over the 1996-2006 and 2007-2017 decades.

To avoid the influence of the seasonal unstationarity, they are presented here only at 8-day time intervals, so, we could not reveal in these statistical parameters the influence of the stratospheric ozone variations over Moscow with periods of several weeks typical for planetary waves. These strong variations observed in winter months as a result of shifts of the stratospheric polar vortex poor in ozone air to Europe and back to the North Pole can be discerned in Figure 3. So, as a subject of future research, the study of time correlation periods of $2-8$ weeks is planned. For this, it will be necessary to distract at calculations seasonal changes of mean ozone profiles.

To explain revealed changes in both ozone profiles and their variances in the stratosphere over Moscow, it is necessary to take into account that the geographical position of Moscow (56N, 37E) allowed observing alternations of air masses of very different origins, especially in periods of violent stratospheric disturbances and stratospheric warmings.

The main factors influencing the ozone profile and its integral content in the stratosphere $(\sim 90 \%$ of the total ozone column content) at time scales of weeks and months are stratospheric dynamics (both large-scale and mesoscale) and photochemistry (especially reactions with ozone-depleting substances). Both of the factors are related to each other, seasonally dependent, and vary considerably from year to year. Dynamics is the most important in the lower and middle stratosphere, while the role of photochemical processes increases in the upper stratosphere and mesosphere. To date, predictions of large-scale dynamical events such as planetary waves, stratospheric warmings, and their inter-annual variations are difficult and unreliable. At time scales of years and decades, the stratospheric ozone is additionally influenced by solar activity, quasi-biennial and other atmospheric and oceanic oscillations, trends in ozone-depleting substances content and spatial distribution, aerosol from volcanic eruptions, etc. [30].

The 1996-2017 ozone observations at the LPI occasionally but almost exactly covered the 23red and 24th 11-year solar cycles (see in Figure 21 the corresponding solar activity dynamics 
according to https://yandex.ru/images/search?text=11-years\%20cycle\%20of\%20solar\%20activity\& stype $=$ image \&lr $=47 \&$ source $=$ wiz\&pos=25\&img_url=https $\% 3 \mathrm{~A} \% 2 \mathrm{~F} \% 2 \mathrm{Fwww} \cdot$ spaceweather.live $\%$ 2Fimages\%2Fnews\%2F400-header.jpg\&rpt=simage).

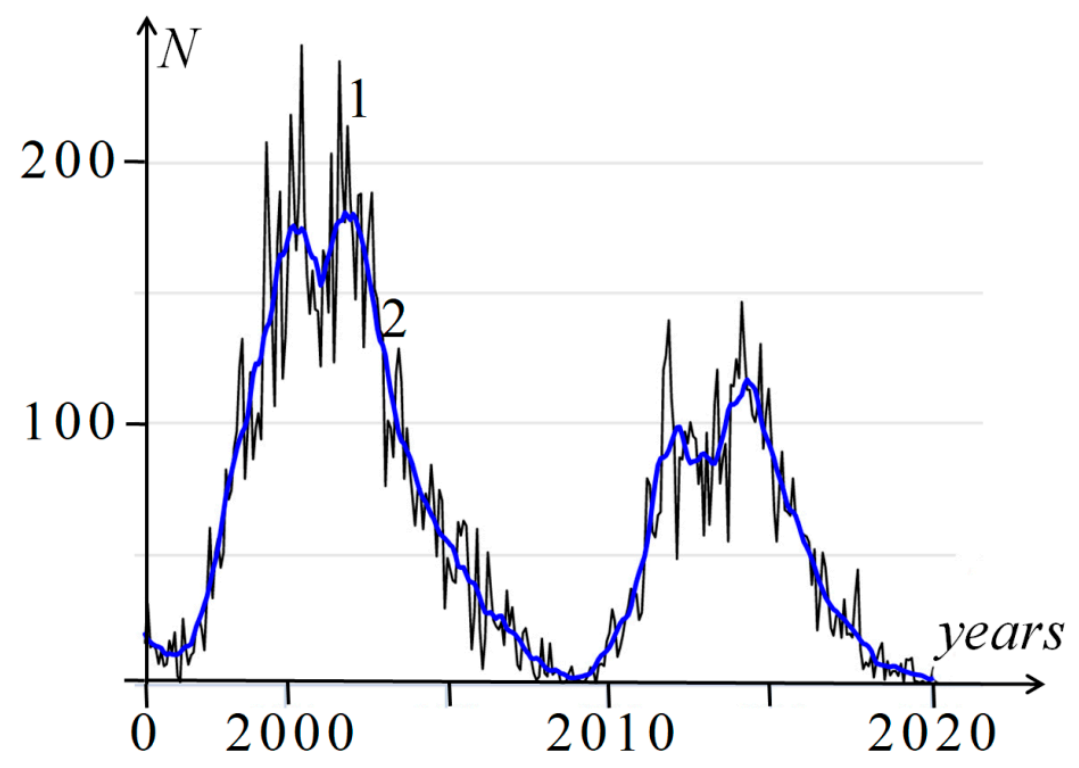

Figure 21. The 23rd and 24th cycles of solar activity: 1-monthly mean number of solar spots; 2-smoothed line.

Expected variations of column ozone content related to changes in solar activity amount to $1-2 \%$ with the maximum in polar latitudes [30]. Solar activity in the 24th cycle was almost twice lower than that in the 23rd one. In the absence of other factors, it should have resulted in a decrease in the mean column ozone content during the 24th cycle in comparison to the $23 \mathrm{rd}$ one. In our measurements, the difference between integral mean ozone content above $20 \mathrm{~km}$ in the decades 2007-2017 and 1996-2006 was small and negative-about -0.7\%. It is most probable that obtained minor differences between the two decades for both the integral mean ozone content above $20 \mathrm{~km}$ and integral mean ozone profile (see Figure 15a) resulted from the cumulative effect of solar activity; trends of ozone depleting substances; and changes in global atmospheric dynamics, composition (increase in $\mathrm{CO}_{2}$ content), and temperature. It should also be mentioned that relative variations of the ozone concentration in the upper stratosphere and lower mesosphere related to solar activity are more significant than those of the corresponding integral ozone content. For example, it is reported that in the 23rd cycle, relative ozone changes between the solar minimum and maximum in the region of the Antarctic ozone hole amounted to $6.8-9.6 \%$ at altitudes of $22-31 \mathrm{~km}$ [31]. So, this effect could be considered as one of reasons for the decrease in variances in the 2007-2017 decade.

Aerosol influence resulted from Eyjafjallajökull eruption in 2010, and wildfires of the last years were negligible-much smaller than the negative contributions after eruptions of El Chichon in 1982 and Pinatubo in 1991. The effect from the last one almost disappeared in 1996 [30].

To explain noticeable and statistically significant decade offsets of monthly mean ozone profiles and their variances as well as of variances of integral mean ozone profiles (see Figures 4-8, Figures 15 and 16), we suppose that these changes are related mostly to long-term changes of large-scale dynamics of the stratosphere. This explanation is based on [32], where the influence of strong sudden stratospheric warmings on the ozone in the middle stratosphere over Moscow was studied for cold seasons (we will call them "winters") from 1995-1996 to 2014-2015. Analysis of several geophysical parameters at a 10 mbar pressure level (altitude about $30 \mathrm{~km}$ ) such as amplitudes of planetary waves $n=1$ and $n=2$, geopotential height in the stratospheric polar vortex center, speed of mean zonal wind at $85^{\circ} \mathrm{N}$ and 
$60^{\circ} \mathrm{N}$, and difference of mean zonal temperatures at 85 and $60 \mathrm{~N}$ allowed the winters to be divided into four groups:

Group I-winters with major mid-winter stratospheric warming in January; in December polar vortex was shifted to Europe.

Group Ia-winters similar to those of Group I, but the mid-winter stratospheric warming occurred in February, approximately a month later than in Group I.

Group II-winters without strong mid-winter stratospheric warming; polar vortex was deep and long-living; its center was close to the North Pole.

Group III-winters with the shallow stratospheric polar vortex and minor stratospheric warming in December.

The distribution of winters is illustrated by Figure 22.

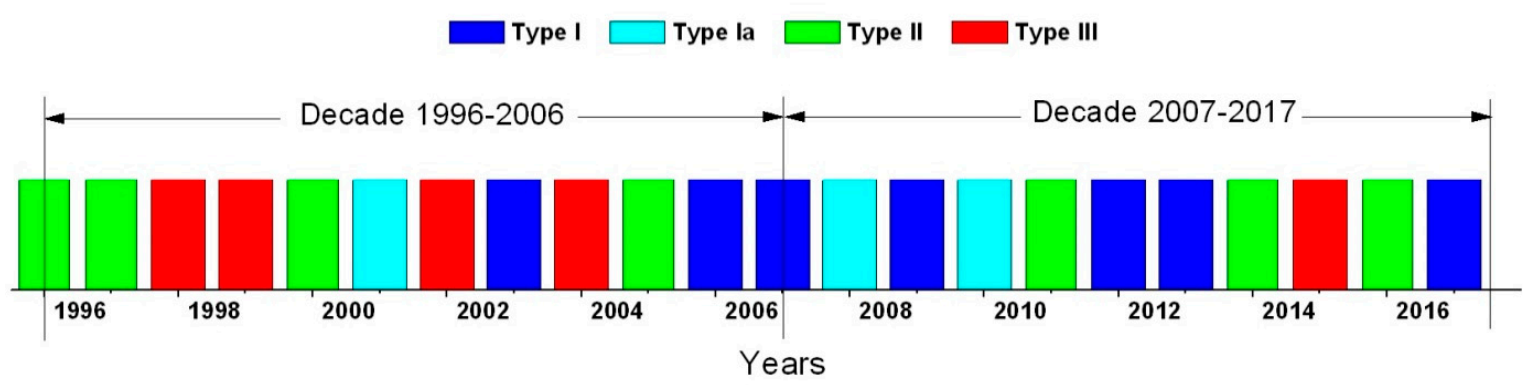

Figure 22. Distribution of the winters from various groups within 1995-2017 period.

Ozone profiles (see Figure 3) were noticeably different for various groups of winters. For Group I, the polar vortex rose rapidly and was often shifted to Europe in November and December, so the zone of low ozone concentration in the vortex typically occurred over Moscow. As a result, ozone mixing ratio fell in December to low values of $u_{\mathrm{I}} \approx 4.5 \mathrm{ppm}$ at the pressure level $10 \mathrm{mbar}$ [32] (herein, we use values averaged through the group of winters). In January, when mid-winter stratospheric warmings occurred, the vortex deformed and filled with rich in ozone air from anticyclone regions. This resulted in a sharp increase up to $u_{\mathrm{I}} \approx 6-6.5$ ppm over Moscow in January and February with subsequent growth up to $\sim 7 \mathrm{ppm}$ in March and April [32].

Otherwise, in winters of Group II, the polar vortex formed a little later and only sometimes was shifted to Europe, so averaged ozone mixing ratio in the stratosphere over Moscow in November and December decreased slower than for Group I; its typical value in December was $u_{\mathrm{II}} \approx 5-5.5 \mathrm{ppm}$ at 10 mbar. This level lasted until the beginning of February, then the ozone concentration grew smoothly up to 6.2-6.7 ppm in March and April. So, in December, the difference $u_{\mathrm{I}}-u_{\mathrm{II}}$ was negative and decreased to $\approx-1 \mathrm{ppm}$. In contrast, in January and at the beginning of February, the difference became positive and reached $1 \mathrm{ppm}$ and above. The resulting span of its variations was typically about 2 ppm [32].

For winters of Group Ia, the variations of ozone concentrations were similar to those of Group I, but transitions from low to high values in the middle stratosphere occurred later, in February. Negative differences of $u_{\mathrm{Ia}}-u_{\mathrm{II}}$ lasted longer (in December and January). Correspondingly, positive values of the $u_{\mathrm{Ia}}-u_{\mathrm{II}}$ difference after the mid-winter stratospheric warming in February were lower and lasted for a shorter time.

In winters of Group III, the main course of ozone changes in the stratosphere over Moscow was similar to that for winters of Group II with irregular but more or less uniform variations of difference $u_{\mathrm{II}}-u_{\mathrm{III}}$ within approximately from -0.6 to $0.8 \mathrm{ppm}$ at $10 \mathrm{mbar}$ level from October to May.

Preliminary estimates of monthly mean ozone variances at $30 \mathrm{~km}$ averaged through winters of the four groups showed that the variances increased from minimum to maximum for winters of Groups I, Ia, II, and III. So, the ozone concentrations in winters of Groups II and III were more volatile, mainly because of multiple shifts of the polar vortex to Europe and back to the North Pole. For example, 
the winter 1996-1997 was the most turbulent one, with 12 shifts of the polar vortex back and forth from November to April. Obvious exceptions from the tendency were January data for winters of Group I and February data for winters of Group Ia-the months when mid-winter stratospheric warmings occurred.

To understand some features of changes in monthly mean ozone profiles and their variances between decades 2007-2017 and 1996-2006 presented in Figures 4-8, Figure 15, and Figure 16, we have to compare numbers of data sets with low or high mean ozone concentration values and with low or high variances for the decades. For example, from Figure 22, one can see that for the decade 1996-2006 in January, there are 2 sets from Group I with a high ozone concentration and 1 set from Group Ia with a low concentration in the middle stratosphere. For the decade 2007-2017, the corresponding numbers are 5 and 2. This means that in January, the mean ozone concentration for the decade 2007-2017 was greater than that for the decade 1996-2006. This could be a reason for the highest maximum in Figure 7a for January at altitudes around $30 \mathrm{~km}$ for the difference between monthly mean ozone profiles for two decades.

Similarly, it is possible to find reasons for changes of monthly mean variances. Note that low-volatile contributions to monthly mean variances originated from winters of Group I (except for January) and Group Ia (except for February); high-volatile ones came from Groups II and III together with January of Group I and February of Group Ia. Using these simple rules, we obtained that the significant increase in the number of low-volatile months and corresponding decrease in the number of high-volatile months for the decade 2007-2017 in December and February resulted in the appearance of two deep minima at altitudes of $30-40 \mathrm{~km}$ in changes of monthly mean variances in Figure $7 \mathrm{~b}$. On the other hand, in January for the decade 1996-2006, the numbers of low- and high-volatile contributions were close to those for the decade 2007-2017. This can explain the small difference between variances of monthly mean ozone profiles in the middle stratosphere in January (see Figure $7 \mathrm{~b}$ ).

These simple examples illustrate the influence of differences in distribution of various winters within the decades on results of statistical analysis.

Taking into account preceding discussion, we can also qualitatively explain noticeable changes in the probability density of ozone profile variations between the decades presented in Figure 4 . The bimodal character of ozone profile variations related to multiple shifts of the polar vortex to Europe and back to the North Pole was typical for winters of groups II and III, which prevailed in the decade 1996-2006 (see Figure 22). So, in the stratosphere over Moscow, the polar vortex poor in ozone air often alternated with rich in ozone air masses outside the vortex. Some hints to this bimodality with spans of 1-2 ppm are seen in Figure $4 \mathrm{a}$ for altitudes of an ozone profile maximum of $30-35 \mathrm{~km}$. Whereas winters of Groups I and Ia prevailing in the decade 2007-2017 (Figure 22) were less volatile, ozone profile variations occurred not so often and their spans were smaller. So, distribution of the probability density of ozone profile variations for the 2007-2017 decade shown in Figure $4 \mathrm{~b}$ is considerably narrower.

\section{Conclusions}

In this paper, the results of the statistical analysis of ensembles of the atmospheric ozone volume mixing ratio profiles over Moscow for 1996-2017 are presented. The profiles were obtained from ground-based day-time microwave measurements of thermal emission in the atmospheric ozone spectral line centered at a frequency of $142.175 \mathrm{GHz}$ carried out at the P.N. Lebedev Physical Institute of the Russian Academy of Sciences for the two decades. Ground-based microwave radiometry is low-dependent on weather conditions in comparison with ground-based optical instruments. It allows day-and-night measurements of the ozone profile from the upper troposphere to the lower mesosphere. So, the method is very effective and suitable for long-term monitoring of the atmospheric ozone profile and its changes. The geographical position of Moscow allows air masses of very different origins to be observed, which leads to strong variations of the ozone profile, especially in periods of violent stratospheric disturbances and stratospheric warmings. Because of these reasons, the results obtained in the same place and with the same method for more than twenty years since the recovery of the 
ozone layer began are very important-especially for the study of long-term changes of the ozone layer. Additionally, the 1996-2017 ozone observations at the LPI almost exactly covered the 23rd and 24th 11-year solar cycles. The important improvement of the ozone data analysis was provided with the use of the proposed method of clearing statistical parameters from the retrieval errors.

Results presented in the paper reveal significant changes in calculated statistical parameters between decades 1996-2006 and 2017-1017 that demonstrate that the evolution of the ozonosphere consists of not only the ozone column change (its decade mean remained practically unchanged) but of multiple seasonal, inter-annual, and more long-term transformations of the ozone profile: strong decade changes in monthly mean variability, altitude distribution of ozone mixing ratio, and space-time correlations.

It was found that relatively stronger decade changes in the monthly mean ozone profiles were observed at altitudes higher and lower than the maximum ozone volume mixing ratio profile. The maximum positive offsets of $13 \%$ of the mean value were discovered at 27 and $44 \mathrm{~km}$ in January, whereas maximum negative changes amounted to 32\% in September and $26 \%$ in May at an altitude of $19 \mathrm{~km}$. The main and unexpected result of this research is the drastically decreased variability of the ozonosphere at altitudes above $30 \mathrm{~km}$, especially in cold months-the decrease in dispersion, inter-altitude, and time variations of the ozone profiles. The maximum fall was detected in February for the monthly mean variances at an altitude of $39 \mathrm{~km}$ in the decade $2007-2017-46 \%$ of those in the decade 1996-2006.

Significant changes were also revealed in distributions of other statistical parameters: probability density of the ozone profile variances, seasonal changes in monthly mean inter-altitude, and time covariance and correlation functions of the ozone profiles-as well as in frequency spectra of these functions.

Furthermore, monthly mean statistical parameters were averaged over all the months from September to May to compare obtained seasonally-independent integral mean parameters for 1996-2006 and 2007-2017 decades. Changes of integral monthly mean ozone profiles appeared considerably smoothed with a small decrease of 2,6\% (with sampling error $1.7 \%$ ) in the profile maximum at $37 \mathrm{~km}$; the maximum became somewhat wider, and its values at lower and higher altitudes were increased by $1.7 \%$ at $28 \mathrm{~km}$ and $2.5 \%$ at $47 \mathrm{~km}$ (with sampling errors of $1.7 \%$ ). Additionally, the integral mean ozone profile decrease amounted to $5.7 \%$ at the altitude of $19 \mathrm{~km}$ (with $1.8 \%$ sampling error). Here, it should be mentioned that the corresponding decade averaged column (integral) ozone content above $20 \mathrm{~km}$ remained practically unchanged $-4.61 \mathrm{~g} / \mathrm{m}^{2}$ for the decade $1996-2006$ versus $4.58 \mathrm{~g} / \mathrm{m}^{2}$ for $2007-2017$.

Integral variance profiles demonstrated the increase at an altitude below $30 \mathrm{~km}$ with the maximum at $23 \mathrm{~km}$ (35\% of the value of variances for 1996-2006 at this altitude) and decrease at altitudes above $30 \mathrm{~km}$ with the maximum at $38 \mathrm{~km}(-25 \%)$. These offsets of variances are statistically significant-like the changes observed in inter-altitude and time covariance functions.

It is possible to conclude that strong decade changes in the ozonosphere revealed in the monthly mean ozone profile are mostly smoothed at the averaging over nine months of analysis, whereas they remain statistically significant for parameters of the profile variations, especially the deep decrease in ozonosphere variability above $30 \mathrm{~km}$ in the cold months of the 2007-2017 decade. As possible factors of this decrease, the transformation of the stratosphere large-scale dynamics between two decades and the simultaneous large reduction in the solar activity in the 2nd decade are proposed and discussed. Furthermore, the contradiction between the decrease in the ozonosphere variability above $30 \mathrm{~km}$ and growth of variability and instability of the atmosphere because of global warming is intriguing and deserves special attention.

Author Contributions: Conceptualization, K.P.G.; Data curation, E.P.K. and S.B.R.; Formal analysis, K.P.G.; Investigation, K.P.G., E.P.K. and S.B.R.; Methodology, K.P.G.; Software, K.P.G. and E.P.K.; Visualization, K.P.G.; Writing-original draft, K.P.G. and S.B.R.; Writing-review and editing, E.P.K. All authors have read and agreed to the published version of the manuscript.

Funding: This research is based on data obtained in 1996-2017, and it received no current external funding. 
Acknowledgments: Authors are very grateful to the British Atmospheric Data Center (BADC) kindly providing the multi-year microwave ozone monitoring at the LPI with meteorological parameters necessary for retrieval and analysis of ozone profiles; to Sergey V. Solomonov, the former Head of the LPI Laboratory of Millimeter-wave Spectroscopy, organizer and team leader of all experimental works used as statistical data in presented here analysis; to Alexandr N. Ignatyev for creation of the LPI ozone profiles data base and highly skilled processing of the BADC data; and to Alexandr N. Lukin who carried out (together with Elena P. Kropotkina) the multi-year ozone observations and performed initial processing of the ozone spectra.

Conflicts of Interest: The authors declare no conflict of interest. The funders had no role in the design of the study; in the collection, analyses, or interpretation of data; in the writing of the manuscript, or in the decision to publish the results.

\section{Appendix A}

\section{Inverse Problem of Microwave Radiometry Ozone Profiling}

After subtraction of the deposition of other atmospheric components, the relation between the brightness temperature $T_{B}$ of thermal radio emission and the profile $U(h)$ of the ozone volume mixing ratio (hereinafter referred to as "ozone") can be written as

$$
T_{B}(f)=\int_{0}^{\infty} U(h) K(U, f, \theta, h) d h,
$$

where $\mathbf{K}=K(U, f, \theta, h)$ is the nonlinear kernel of the equation; $f$ is the frequency; and $\theta$ is the elevation angle of observations. Taking into account measurement errors, the Equation (A1) is solved by data vector $T_{B}^{\delta}=T_{B}+\delta T_{B}$ with errors instead of the exact data vector $T_{B}$. To solve this equation, we used the iteration algorithm based on [16], where at each step of the iteration process, a solution is found by Tikhonov's method of generalized discrepancy [17]. At the first step of iterations, an arbitrary continuous ozone profile $U_{1}(h)$ is substituted into the kernel of (A1); at the second step, the retrieved profile obtained at the first step is substituted into the kernel, and so on. The operator form of this iteration scheme for the nonlinear problem can be written as follows:

$$
\mathbf{K}^{\mathbf{i}} U^{i+1}=T_{B^{\prime}}^{\delta}
$$

where the operator $\mathbf{K}^{\mathrm{i}}=\mathbf{K}(U i, f, \theta, h)$ and the model ozone profile is the first approximation $U_{1}(h)$, which can be chosen as an arbitrary function from $W_{2}^{1}$. The data vector $T_{B}^{\delta}$ is measured with errors that satisfy

$$
\delta T_{B}^{2} \leq \sup \left\|\mathbf{K} U-T_{B}^{\delta}\right\|_{L_{2}}^{2}=\sup \left(\frac{1}{\Delta f} \int_{\Delta f}\left[T_{B}(f)-T_{B}^{\delta}(f)\right]^{2} d f\right) .
$$

Here, $L_{2}$ is the space of square-integrable functions, and $W_{2}^{1}$ is the space of square-integrable functions having square-integrable generalized derivatives. The notation $\|x\|$ means the norm of the function $x$ as an element of the function spaces $\mathrm{L}_{2}$ or $\mathrm{W}_{2}^{1}$. In (A3), $U$ is the exact solution, and $\Delta f$ is the width of the frequency band. In frameworks of this method, the error in the kernel can be taken into account. It can include a discretization error, possible inaccuracy in describing the absorption coefficient, errors related to uncertainty of the temperature, and pressure variations and errors due to nonlinearity of the kernel (in fact, the pressure profile is determined by the temperature profile). In solving the inverse problem with this technique, these profiles were kindly made available to us by the British Atmospheric Data Center (BADC, [1]). The measure of error in the kernel of (A1) is determined as

$$
\delta_{h}^{2} \leq \sup \left\|\mathbf{K}_{h} U-\mathbf{K} U\right\|_{L_{2}}^{2}=\sup \left\|\mathbf{K}_{\mathbf{h}} U-T_{B}\right\|_{L_{2}}^{2}=g^{2}\|U\|_{W_{2}^{1}}^{2}
$$

where $g$ is a constant, and $\mathbf{K}_{h}$ is the operator corresponding to a preset approximate kernel in (2). The above errors can result in the incompatibility of the data with the equation being solved, because a 
smoothing property of the kernel of (A1) limits the class of possible functions $T_{B}(f)$ so that the function $T_{B}^{\delta}$ can fall outside this admissible class in the presence of a random error. Of course, the measure of incompatibility $\delta \mu$ cannot exceed the sum of kernel and measurement errors-i.e.,

$$
\delta_{\mu}=\inf \left\|\mathbf{K}_{\mathbf{h}} U-T_{B}^{\delta}\right\|_{L_{2}} \leq \delta T_{B}+\delta_{h}
$$

According to Tikhonov's method [17], an approximate solution $U_{\alpha}^{i+1}$ minimizes the smoothing functional

$$
\mathbf{M}^{\alpha}\left(U^{i+1}\right)=\left\|\mathbf{K}_{h}^{i} U^{i+1}-T_{B}^{\delta}\right\|_{L_{2}}^{2}+\alpha\left\|U^{i+1}\right\|_{W_{2}^{1}}^{2}
$$

In particular, for the smoothing functional (6), we have

$$
\left\|U^{i+1}\right\|_{W_{2}^{1}}^{2}=\frac{1}{\Delta h} \int_{h_{\min }}^{h_{\max }}\left\{\left[U^{i+1}(h)\right]^{2}+\left[\Delta h \frac{d U^{i+1}}{d h}\right]^{2}\right\} d h,
$$

where $\Delta h=h_{\max }-h_{\min }$ is the altitude interval on which the solution is sought.

The regularization parameter $\alpha$ that determines smoothing of the approximate solution is expressed by the root of the one-dimensional nonlinear equation of generalized discrepancy, which takes into account all the above components of the total error:

$$
\rho(\alpha)=\left\|\mathbf{K}_{h}^{i} U_{\alpha}^{i+1}-T_{B}^{\delta}\right\|_{L_{2}}^{2}-\delta^{2}=0 .
$$

where $U_{\alpha}^{i+1}$ is the function (solution) that minimizes functional (A6), and $\delta^{2}=\left(\delta T_{B}+\delta_{h}\right)^{2}+\delta_{\mu}^{2}$ is the effective error parameter.

This parameter includes all sources of errors: measurement errors, the inaccuracy in describing the kernel, and the measure of incompatibility of Equation (A2) with its right-hand side. The regularization parameter $\alpha$ and, consequently, the degree of smoothness of the approximate solution, are determined by this error parameter. Here, it is worth mentioning that the definition of $\delta$ is not unique-its choice can depend on goals of every application. If we want to retrieve the small-scale structure of a solution, then the values of $\delta$ should be relatively small, but some artifacts can appear; if we want to avoid artifacts, the value of $\delta$ should be increased, but as a result, we can obtain a too smoothed solution. In our statistical studies in this paper, the choice is based on minimization of rms errors of retrieval, which is a compromise between these two strategies.

In the method of generalized discrepancy, when data errors tend to zero in the integral metric $\mathrm{L}_{2}$, the approximate solution converges to the exact solution in the metric $W_{2}^{1}$ [17]. It is the main advantage of this method over other known methods. This means that, according to the Sobolev's imbedding theorem, this approximate solution converges uniformly-i.e., in the metric $\mathrm{C}$, where the maximum modulus is the norm. On the other hand, unlike well-posed problems, the rate of convergence here is not proportional to a decrease in $\delta$, but it is slower-as in all ill-posed problems. If the metric $\mathrm{L}_{2}$ (square integrability) is used in the second term of (6), the convergence of the solution will also be in the metric $\mathrm{L}_{2}$ [17].

The parameters $\delta_{h}$ and $\delta_{\mu}$ can he determined from the numerical simulation in the course of minimizing (6). The measure of incompatibility and the degree of kernel nonlinearity limit the level of discrepancy up to which functional (6) can be minimized. Once corresponding discretization has been performed, the problem of minimization of a smoothing functional is transferred to its finite-dimensional analogue representing the well-known quadratic programming algorithm (see [17]).

In solving nonlinear problems, the iteration procedure converges to the exact solution if the conditions of the principle of contracting mappings are fulfilled-i.e., if a completely continuous operator (A1) maps a closed convex set of Banach space onto itself. Because the problem under consideration was solved numerically, the convergence conditions were studied in numerical experiments. 
To minimize a systematic error in the measured brightness temperature related to the calibration, we transform the solving algorithm (A2) to the equation that is mathematically equivalent to (A1):

$$
\begin{gathered}
T_{B}^{\prime}(f)=\int_{0}^{\infty} U^{i+1}(h) K^{\prime}\left(U^{i}, f, \theta, h\right) d h, \\
T_{B}^{\prime}=T_{B}^{\delta}(f)-T_{B}^{\delta}\left(f_{0}\right), K^{\prime}\left(U^{i}, f, \theta, h\right)=K\left(U^{i}, f, \theta, h\right)-K\left(U^{i}, f_{0}, \theta, h\right),
\end{gathered}
$$

where $f_{0}$ is a fixed frequency.

A general property of the Fredholm Equation (A1) as a typical ill-posed problem consists of the lack of a definite relation between the error of data and the accuracy of retrieval because it depends significantly on the shape of the function under retrieval. Therefore, a study of the retrieval accuracy in relation to measurement errors, the number and frequencies of the spectral channels, etc., can be performed through numerical simulations and in no other way.

In applying the method under consideration, it is necessary to define the relation between the effective error parameter $\delta$ given in (A8) and available characteristics of measurement errors. If the errors have a normal distribution with the mean value $\Delta T_{B}(f)$ and standard deviation $\sigma_{T_{B}}(f)$, the error $\delta T_{B}$ can be determined as the mean-square value of the integral in (3) rather than its maximum value. Then, we have

$$
\delta T_{B}^{2}=\frac{1}{\Delta f} \int_{\Delta f}\left\langle\left[T_{B}(f)-T_{B}^{\delta}(f)\right]^{2}\right\rangle d f=\frac{1}{\Delta f} \int_{\Delta f}\left[\sigma_{T B}^{2}(f)+\Delta T_{B}^{2}(f)\right] d f .
$$

It is seen that the determined error is split into the random and systematic terms. In our case, the first term of (A11) is determined by the radiometer noise, whereas the second one includes errors of the absolute calibration and apparatus function. The numerical simulation of this method included:

(a) Formation of the simulated ozone profile;

(b) Computations of the spectrum of brightness temperature;

(c) Adding of simulated Gaussian random errors with zero mean value $\Delta T_{B}(f)$ and preset mean-square variations $\sigma_{T}^{2}(f)$;

(d) Solving the inverse problem (9);

(e) Comparison of the retrieved and simulated ozone profiles.

The error component $\delta_{h}$ caused by the kernel uncertainty due to discretization was minimized down to the level $\delta_{h}=0.001 \mathrm{~K}$ that was well below the radiometer noise $\delta T_{B}$. The value of the kernel error can be considered as the accuracy limit of the numerical scheme that can be implemented with our technique. The kernel uncertainty due to errors in the temperature profile that can be comparable to $\delta T_{B}$ should be also taken into account in $\delta_{h}$. Unfortunately, we do not have enough statistical information about errors in the temperature profile, so it is difficult to optimize the algorithm relative to the parameter $g$ in (A4) especially in the statistical study. The estimation of the measure of incompatibility $\delta_{\mu}$ at the minimization of (6) showed that nonlinearity of the kernel does not lead to an incompatibility with the spectrum of brightness temperatures at each step of iterations. Thus, it follows from (A5) that $\delta_{\mu}<\delta T_{B}$ in the algorithm under consideration. However, taking into account the deposition of temperature errors, we set:

$$
\delta_{\mu}=\delta T_{B}
$$

Thus, the total error $\delta$, with possible incompatibility taken into consideration, must be

$$
\delta=\sqrt{2} \delta T_{B}=\sqrt{2} \sigma_{T_{B}}
$$


Again, we note that $\delta$ is a rather free parameter that depends on the chosen retrieval strategy. If one assumes lower values of $\delta$, there is a probability to obtain peculiarities of the retrieved profile that do not exist in reality. In contrast, if a too enhanced $\delta$ is used in solving, actual features of the preset profile can be smoothed. Thus, the parameter $\delta$ in (A13) can be considered as a desired optimum obtained in numerical experiments.

The numerical simulation was carried out for ground-based observations of ozone layer at the LPI by data of radiometer measurements. The assumed $r m s$ parameter $\delta T_{B}=0.05 \mathrm{~K}$ is achieved in the LPI radiometer at the signal integration time of order $1 \mathrm{~h}$.

In numerical simulations $[9,23]$, the above-mentioned property of uniform convergence of the method have been used. Owing to this property, the maximum modulus of the difference between numerically retrieved and simulated ozone profiles can be considered as a measure of the solution accuracy. Because the quality of a solution depends on complexity of a profile, it was reasonable to obtain estimates of retrieval accuracy for both typical and extreme profiles. Results of the numerical simulation $[9,16]$ demonstrate that errors of retrieved profiles in the altitude range from 20 to $50 \mathrm{~km}$ do not exceed $2-3 \%$ of ozone profile maxima.

This estimation should be, in fact, considered as a minimal error level of this method. In real conditions, there are also errors related to inaccuracy of preset temperature and pressure profiles in the kernel (up to 2.5 and $1.5 \%$, respectively), calibration errors (about $1 \%$ ), and apparatus function distortions (1\%). As a result, the real accuracy of retrieval in the $20-50 \mathrm{~km}$ region could enhance up to 5-7\%; at $15-20 \mathrm{~km}$ and 50-70 km, it can exceed monthly mean ozone $r m s$ variances. However, effects of retrieval errors can be effectively suppressed in statistical parameters of retrieved profiles (sampling random errors of mean values are reduced as $1 / \sqrt{N}$, ( $N$-number of elements in ensembles); moreover, the variances can be cleared from retrieval errors with the method proposed below, systematic errors are eliminated in time correlation parameter, and it is reasonable to suppose that all kinds of errors have no such time evolution as detected seasonal and decade changes of ozonosphere parameters.

At this point, when radiometric measurements of the ozone profile in LPI formed representative statistical ensembles for various seasons, it appeared possible to obtain rms estimations of the accuracy of the method [10], using elements of ensembles as simulated ozone profiles in the numerical simulation of their retrieval at the same level of simulated random errors. Results show that rms errors of retrieved profiles of 0.1-0.3 ppm (depending on the ensemble choice) are quite stable in the altitude region of $15-80 \mathrm{~km}-$ i.e., $2-5 \%$ of mean ozone mixing ratio values in the maximum of the ozone layer in the $20-50 \mathrm{~km}$ region. As mentioned above, it is also necessary to take into account that the real level of errors mostly related to unaccounted temperature errors can enhance up to $0.4-0.5 \mathrm{ppm}$. However, corresponding retrieval errors also are suppressed in the studied statistical parameters and hardly have a significant effect on the revealed changes of ozone statistics.

\section{References}

1. SPARC/IO3C/GAW Report on Long-Term Ozone Trends and Uncertainties in the Stratosphere; SPARC Report No. 9; Petropavlovskikh, I.; Godin-Beekmann, S.; Hubert, D.; Damadeo, R.; Hassler, B.; Sofieva, V. (Eds.) SPARC: Richmond, VA, USA, 2019. Available online: http://www.sparc-climate.org/publications/sparc-reports (accessed on 10 October 2020). [CrossRef]

2. Steinbrecht, W.; Froidevaux, L.; Fuller, R.; Wang, R.; Anderson, J.; Roth, C.; Bourassa, A.E.; Degenstein, D.A.; Damadeo, R.; Jones, N.B.; et al. An update on ozone profile trends for the period 2000 to 2016. Atmos. Chem. Phys. 2017, 17, 10675-10690. [CrossRef]

3. Manney, G.L.; Lawrence, Z.D.; Santee, M.L.; Livesey, N.J.; Lambert, A.; Pitts, M.C. Polar processing in a split vortex: Arctic ozone loss in early winter 2012/2013. Atmos. Chem. Phys. 2015, 15, 5381-5403. [CrossRef]

4. Tao, M.; Konopka, P.; Ploeger, F.; Grooß, J.-U.; Müller, R.; Volk, C.M.; Walker, K.A.; Riese, M. Impact of the 2009 major sudden stratospheric warming on the composition of the stratosphere. Atmos. Chem. Phys. 2015, 15, 8695-8715. [CrossRef] 
5. Smyshlyaev, S.P.; Pogorel'tsev, A.I.; Galin, V.Y.; Drobashevskaya, E.A. Influence of wave activity on the composition of the polar stratosphere. Geomagn. Aeron. Engl. Transl. 2016, 56, 95-109. [CrossRef]

6. Wang, W.; Tian, W.; Dhomse, S.; Xie, F.; Shu, J.; Austin, J. Stratospheric ozone depletion from future nitrous oxide increases. Atmos. Chem. Phys. 2014, 14, 12967-12982. [CrossRef]

7. Manney, G.L.; Santee, M.L.; Rex, M.; Livesey, N.J.; Pitts, M.C.; Veefkind, P.; Nash, E.R.; Wohltmann, I.; Lehmann, R.; Poole, L.R.; et al. Unprecedented Arctic ozone loss in 2011. Nature 2011, 478, 469-475. [CrossRef]

8. Kulikov, U.U.; Markina, N.N.; Naumov, A.P.; Riskin, V.G.; Sumin, M.I. Retrieval of Height Ozone Distribution by Ground-Based Measurements of Integral Absorption in the Millimeter Range. Atmos. Ocean. Phys. 1988, $24,1282-1292$.

9. Gaikovich, K.P.; Kropotkina, E.P.; Solomonov, S.V. Vertical ozone profile determination from ground-based measurements of atmosphere millimeter-wave radiation. Izv. Atmos. Ocean. Phys. 1999, 35, 78-86.

10. Solomonov, S.V.; Gaikovich, K.P.; Kropotkina, E.P.; Rozanov, S.B.; Lukin, A.N.; Ignat'ev, A.N. Remote sensing of atmospheric ozone at millimeter waves. Radiophys. Quantum Electron. 2011, 54, 102-110. [CrossRef]

11. Fernandez, S.; Rufenacht, R.; Kampfer, N.; Portafaix, T.; Posny, F.; Payen, G. Results from the validation campaign of the ozone radiometer GROMOS-C at the NDACC station of Reunion island. Atmos. Chem. Phys. 2016, 16, 7531-7543. [CrossRef]

12. Kropotkina, E.P.; Rozanov, S.B.; Lukin, A.N.; Ignat'ev, A.N.; Solomonov, S.V. Characteristics of Changes in the Ozone Content in the Upper Stratosphere over Moscow during the Cold Half-Years of 2014-2015 and 2015-2016. Geomagn. Aeron. 2017, 57, 361-368. [CrossRef]

13. Solomonov, S.V.; Rozanov, S.B.; Kropotkina, E.P.; Lukin, A.N. A millimeter-wave spectroradiometer for the remote sensing of atmospheric ozone. J. Commun. Technol. Electron. 2000, 45, 1377-1382.

14. Solomonov, S.V.; Ignat'ev, A.N.; Kropotkina, E.P.; Logvinenko, S.V.; Lukin, A.N.; Nikiforov, P.L.; Rozanov, S.B. Spectral instrumentation for monitoring atmospheric ozone at millimeter waves. Instrum. Exp. Tech. 2009, 52, 280-286. [CrossRef]

15. Solomonov, S.V.; Kropotkina, E.P.; Lukin, A.N.; Ponomarenko, N.I.; Rozanov, S.B.; Ellder, J. Some features of the vertical ozone distribution from millimeter wave measurements at Pushchino and Onsala observatories. J. Atmos. Terr. Phys. 1994, 56, 9-15. [CrossRef]

16. Gaikovich, K.P. Tikhonov's Method of the Ground-based Radiometric Retrieval of the Ozone Profile, IGARSS'94 Digest. In Proceedings of the IGARSS'94-1994 IEEE International Geoscience and Remote Sensing Symposium, Pasadena, CA, USA, 8-12 August 1994; Volume 4, pp. 1901-1903.

17. Tikhonov, A.N.; Arsenin, V.Y. Solutions of Ill-Posed Problems; Winston: New York, NY, USA, 1977.

18. Chahine, M.T. Inverse Problems in Radiative Transfer: Determination of Atmospheric Parameters. J. Atmos. Sci. 1970, 27, 960-967. [CrossRef]

19. Waters, J.W.; Hardy, J.O.; Jarnot, R.F.; Picket, H.H. Chlorine Monoxide Radical, Ozone and Hydrogen Peroxide. Science 1981, 214, 61-64. [CrossRef]

20. Rodgers, C.D. Characterization and Error Analyses of Profiles Retrieved from Remote Sensing Measurements. J. Geophys. Res. D Atmos. 1990, 95, 5587-5595. [CrossRef]

21. Brillet, J. A Theoretical Study of Ozone Measurements Made with Ground-based Microwave Sensors. J. Geophys. Res. D Atmos. 1989, 94, 12833-12850. [CrossRef]

22. Gaikovich, K.P.; Kitaj, S.D.; Naumov, A.P. On Determination of Ozone and Other Minor Gases of the Atmosphere by Limb-Viewing Measurements from Satellites in the Microwave Range. Russ. J. Remote Sens. 1991, 3, 73-81.

23. Gaikovich, K.P. Inverse Problems in Physical Diagnostics; Nova Science Publishers Inc.: New York, NY, USA, 2004.

24. Scientific Assessment of Ozone Depletion: 2018, World Meteorological Organization, Global Ozone Research and Monitoring Project-Report No.58, Geneva. 2018. Available online: https://www.esrl.noaa.gov/csl/ assessments/ozone/2018/ (accessed on 10 October 2020).

25. Froidevaux, L.; Livesey, N.J.; Read, W.G.; Jiang, Y.B.; Jimenez, C.; Filipiak, M.J.; Schwartz, M.J.; Santee, M.L.; Pumphrey, H.C.; Wu, D.L.; et al. Early validation analyses of atmospheric profiles from EOS MLS on the Aura satellite. IEEE Trans. Geosci. Remote Sens. 2006, 44, 1106-1121. [CrossRef]

26. Rozanov, S.B.; Solomonov, S.V.; Kropotkina, E.P.; Ignatyev, A.N.; Lukin, A.N. Ground-based remote sensing of the atmospheric ozone over Moscow at millimeter waves. Proc. SPIE 2006, 6362, U472-U482. 
27. Rozanov, S.B.; Zavgorodniy, A.S.; Solomonov, S.V.; Kropotkina, E.P.; Lukin, A.N.; Ignatyev, A.N. Microwave measurements of stratospheric and mesospheric ozone in Moscow. In Proceedings of the IGARSS 2018 IEEE International Geoscience and Remote Sensing Symposium, Valencia, Spain, 22-27 July 2018; pp. 3116-3119.

28. Moreira, L.; Hocke, K.; Eckert, E.; von Clarmann, T.; Kämpfer, N. Trend analysis of stratospheric ozone profiles observed by GROMOS microwave radiometer at Bern. Atmos. Chem. Phys. 2015, 15, 10999-11009. [CrossRef]

29. Scientific Assessment of Ozone Depletion: 2014, World Meteorological Organization, Global Ozone Research and Monitoring Project-Report No.55, Geneva. 2014. Available online: https://www.esrl.noaa.gov/csl/ assessments/ozone/2014 (accessed on 10 October 2020).

30. Zvyagintsev, A.M.; Vargin, P.N.; Peshin, S. Total ozone variations and trends during the period 1979-2014. Atmos. Ocean. Opt. 2015, 28, 575-584. [CrossRef]

31. Grytsai, A.; Evtushevsky, O.; Klekociuk, A.; Milinevsky, G.; Yampolsky, Y.; Ivaniha, O.; Wang, Y. Investigations of the vertical influence of the 11-year solar cycle on ozone using SBUV and Antarctic ground-based measurements and CMIP6 forcing data. Atmosphere 2020, 11, 873. [CrossRef]

32. Solomonov, S.V.; Kropotkina, E.P.; Rozanov, S.B.; Ignat'ev, A.N.; Lukin, A.N. Influence of strong sudden stratospheric warmings on ozone in the middle stratosphere according to millimeter wave observations. Geomagn. Aeron. 2017, 57,361-368. [CrossRef]

Publisher's Note: MDPI stays neutral with regard to jurisdictional claims in published maps and institutional affiliations. 